\title{
Does ambiguity aversion survive in experimental asset markets?
}

\author{
Sascha Füllbrunn, Holger Rau†, and Utz Weitzel ${ }^{\ddagger \S}$
}

March 27, 2014

\begin{abstract}
Although a number of theoretical studies explain empirical puzzles in finance with ambiguity aversion, it is not a given that individual ambiguity attitudes survive in markets. In fact, despite ample evidence of ambiguity aversion in individual decision making, most studies find no or only limited ambiguity aversion in experimental financial markets, even when they exclude arbitrage. We argue that ambiguity effects in markets depend on market feedback and on a sufficiently strong bias toward ambiguity among the participants. Accordingly, we find significant ambiguity effects in lowfeedback call markets for assets that provoke high ambiguity aversion, but no ambiguity effects in high-feedback double auctions.
\end{abstract}

\footnotetext{
*Radboud University Nijmegen, IMR, Department of Economics, Thomas van Aquinostraat 5, 6525 GD Nijmegen, The Netherlands

${ }^{\dagger}$ Universität Erlangen-Nürnberg, Lehrstuhl für Volkswirtschaftslehre, insbesondere Wirtschaftstheorie, Lange Gasse 20, 90403 Nürnberg, Germany

${ }^{\ddagger}$ Radboud University Nijmegen, IMR, Department of Economics, Thomas van Aquinostraat 5, 6525 GD Nijmegen, The Netherlands (u.weitzel@fm.ru.nl)

$\S$ We thank seminar participants at the ESA World Meeting in New York (2012), the Experimental Finance Conference in Luxembourg (2012), and the ESA European Meeting in Cologne (2012) for helpful comments. All remaining errors are ours.
} 


\section{Introduction}

Many real-life decisions are characterized by ambiguity, in which we lack important information such as the objective probabilities of the relevant states. Keynes (1921) proposed a simple thought experiment to illustrate the effects of ambiguity.

Imagine "[...] the two cases following of balls drawn from an urn. In each case we require the probability of drawing a white ball; in the first case we know that the urn contains black and white in equal proportions; in the second case the proportions of each color is unknown, and each ball is as likely to be black as white. It is evident that in either case the probability of drawing a white ball is $1 / 2$, but that the weight of the argument in favor of this conclusion is greater in the first case." (Keynes, 1921, Chapter VI.6) ${ }^{1}$

Ellsberg (1961) used this experimental design, commonly referred to as the '2color Ellsberg urn' to show that a preference for the risky urn (with measurable probabilities) over the ambiguous urn (with immeasurable probabilities) violates the Subjective Expected Utility Theory and the Sure-thing Principle of the Savage axioms (Savage, 1954). Since then a large body of individual choice experiments have confirmed that, on average, decision makers are 'ambiguity averse' when confronted with the above-quoted choice? In a recent survey of the experimental literature, Trautmann and Van De Kuilen (forthcoming) conclude that "there is clear evidence that on the average, and across various elicitation methods, ambiguity aversion is the typical qualitative finding."

Ambiguity aversion is a possible cause for a number of empirical puzzles in financial economics, which expected utility theory would consider to be (behavioral) anomalies. After the development of several non-expected utility models of individual decision making that considered ambiguity attitudes (e.g., Gilboa and Schmeidler, 1989, Ghirardato et al., 2004, Klibanoff et al., 2005; Nau, 2006), a growing number of theoretical papers incorporated ambiguity aversion into market models to explain long-standing anomalies in finance, like the equity premium puzzle (Epstein and Wang, 1994, Maenhout, 2004, Cao et al., 2005; Leippold et al. 2008), portfolio inertia (Epstein and Wang, 1994, Illeditsch, 2011), the familiarity bias and the home bias in investments (Uppal and Wang 2003. Huang, 2007, Cao et al. 2011), amplification effects (Routledge and Zin,

1 Keynes (1921) did not use the term ambiguity. Instead, he referred to 'the weight of arguments', but was not sure about this concept. In fact, at the beginning of the Chapter VI he writes: "[A]fter much consideration I remain uncertain as to how much importance to attach to it" (Keynes, 1921 Chapter VI.1).

${ }^{2}$ For excellent overviews, see Camerer and Weber (1992), Wakker (2010), Etner et al. (2012), and Trautmann and Van De Kuilen (forthcoming). 
2009, Guidolin and Rinaldi, 2010, Illeditsch, 2011), and asymmetric reactions to good and bad news (Epstein and Schneider, 2008, Epstein et al., 2010; Illeditsch, 2011).

Yet, it is not a given that ambiguity aversion found in individual decision making arises and even survives when market forces are at work. In markets, decisions are no longer independent as they are subject to market feedback from other traders. According to the efficient market hypothesis (henceforth EMH, Fama, 1970), market mechanisms and incentives should eliminate or at least reduce behavioral biases and non-expected utility behavior, including ambiguity aversion (Camerer, 1987). In fact, despite the burgeoning theoretical literature and the promising explanatory potential of ambiguity aversion, no experimental study has provided definite evidence of ambiguity aversion in experimental asset markets (see section 2.1).

This study attempts to shed some light on the underlying reasons for the discrepancy between ambiguity effects in individual decision making and experimental asset markets ${ }^{3}$ In doing so we propose and test an experimental setup in which we give ambiguity effects ample room to survive market forces. In our search for possible underlying reasons, we start with the conditions of the EMH under which (efficient) market forces can eliminate ambiguity effects. The EMH rests on three, progressively weaker conditions, any one of which will lead to market efficiency: (i) full rationality, (ii) independent deviations from rationality, and (iii) arbitrage (Shleifer, 2003). We reformulate these three conditions for market efficiency under ambiguity as follows: either (i) all market participants are ambiguity neutral; or if (i) does not hold, (ii) a symmetric distribution of ambiguity attitudes around ambiguity neutrality offsets the influence of ambiguity-averse and ambiguity-seeking market participants; or if both (i) and (ii) do not hold, (iii) ambiguity-neutral traders take full advantage of arbitrage opportunities.

Condition (i) can be ruled out by clear experimental evidence that individuals frequently violate ambiguity neutrality (see section 2). In many experiments, condition (iii) also does not apply because even in the absence of arbitrage opportunities ambiguity aversion did not survive in experimental asset markets (see section 2.1). However, in support of condition (ii), a number of recent studies have found that the distribution of ambiguity attitudes is often quite symmetric around neutrality (Halevy, 2007, Corgnet et al., 2013) including ambiguity seekingness (e.g., Einhorn and Hogarth, 1985, Kahn and Sarin, 1988, Curley and Yates, 1989) and a large percentage of people with highly inconsistent behavior

\footnotetext{
${ }^{3}$ With ambiguity effects we generally refer to differences between, ceteris paribus, ambiguous and risky assets with regard to asset prices, volatility, trading volume or final asset holdings.
} 
under ambiguity (Charness et al., 2013). In this paper, we therefore focus on condition (ii) for two possible explanations of the empirical phenomenon that ambiguity effects are rarely found in experimental asset markets.

For our first explanation, the group of people with inconsistent ambiguity attitudes is of special interest. Experimental evidence on group behavior and feedback shows that ambiguity neutrality has a 'persuasive edge' with a tendency to de-bias ambiguity-seeking and ambiguity-averse individuals (Charness et al. 2013 Keck et al. 2011). Market participants also use market prices to update heterogeneous beliefs (Banerjee, 2011) and to coordinate price forecasts (Hommes, 2011). We therefore conjecture that continuous market feedback may de-bias participants with inconsistent ambiguity attitudes and may serve as a reason why ambiguity aversion is rarely observed in experimental asset markets. To test our hypothesis on market feedback we compare the ambiguity premium, i.e., the price difference between the risky asset and the ambiguous asset between two market institutions: a continuous open-book double auction with a high level of intra-period market feedback and a call market without any intra-period market feedback $4^{4}$ Note that the current literature primarily administers double auction markets. We suggest that this market institution provides sufficient feedback to wash out ambiguity effects.

Our second explanation builds on evidence that ambiguity attitudes are very heterogeneous and that the bias of the distribution toward ambiguity aversion is, even in individual decision making, not very strong and is sometimes close to neutral (Trautmann and Van De Kuilen, forthcoming). Based on the divergence of opinions literature, which posits that optimists determine asset prices even if they are in the minority (Miller, 1977), it is possible that the average bias toward ambiguity aversion is not strong enough for ambiguity premiums to survive in markets. To investigate the validity of this argument we increase the ambiguity aversion in a market. We do this by comparing a medium objective winning probability condition (50\%) to a high objective probability condition $(75 \%)$ in a 2-color Ellsberg urn setup. This setup has already been proven to increase average ambiguity aversion in individual decision-making tasks (e.g., Abdellaoui et al. 2011). By applying this setup to ambiguous assets in markets, we expect to observe only a small or a zero ambiguity premium in markets with a medium winning probability but stronger ambiguity effects for assets with a high winning probability.

Our results suggest that limited market feedback (call market) in combination with a high winning probability $(75 \%)$ leads to a significant ambiguity premium. Under this condition we observe further ambiguity effects such that for the ambiguous asset the number of executed trades is higher and final asset

\footnotetext{
${ }^{4}$ In the call market traders are informed of the clearing price after the period.
} 
holdings are more dispersed. With double auction markets we find no evidence that ambiguity effects occur under any winning probability, and we find no evidence that ambiguity effects occur under a medium winning probability independent of the market institution 5 In response to our question stated in the title, we conclude that ambiguity aversion can indeed arise and survive in markets. But this statement only holds if market feedback is limited and if the distribution of ambiguity attitudes among traders is sufficiently biased toward ambiguity aversion.

Our paper contributes to this literature by providing clear evidence of ambiguity effects in markets. Most importantly, it identifies two crucial antecedents for their existence: the type of market institution and the distribution of ambiguity attitudes. In doing so, we add to the literature by not only considering the ambiguity effects within certain conditions (market institution, objective winning probability) but also by directly comparing the effects of ambiguity across conditions (call market vs. double auction, medium vs. high objective winning probability).

\section{Theoretical and experimental background}

\subsection{Ambiguity effects in experimental asset markets}

Table 1 provides an overview of experimental asset market studies comparing risky with ambiguous assets. To the best of our knowledge, Camerer and Kunreuther (1989) were the first to study the ambiguity effects in experimental markets. They administered open outcry double auction markets for insurance coverage against risky and ambiguous hazards that produced only losses. The markets were separated without opportunities for arbitrage between risk and ambiguity. The authors conclude that "the effects of ambiguity are rather minor and mixed" (p.287), with conflicting effects on the number of insurance contracts held at the end of trading and no effects on prices.

Sarin and Weber (1993) explored a multitude of combinations of treatment effects, including uniform price auctions vs. open outcry double auctions, independent vs. simultaneous trading of risky and ambiguous assets (with arbitrage), experienced executives vs. students as traders, smaller vs. larger number of trading periods, low vs. equal winning probabilities, and the framing of ambiguity as nature vs. expert judgments. In all treatments, compound lotteries were used to operationalize ambiguity. The study of Sarin and Weber (1993) is demonstrative as the authors administered only one market per condition

\footnotetext{
${ }^{5}$ In a robustness check using the same source of ambiguity we find significant ambiguity premiums in a Becker-DeGroot-Marschak individual decision-making task (Becker et al. 1964).
} 
and report their results graphically. Market prices for ambiguous assets appear to be below prices for risky assets in almost each period for a winning probability of $50 \%{ }^{6}$ Note, that this result is based on only two observations with simultaneous trading of risky and ambiguous assets, which up to now have constituted the entire experimental evidence on the simultaneous trading of risky and ambiguous shares with independent outcomes.

Bossaerts et al. (2010) used a double auction environment to experimentally investigate the simultaneous trading of three assets with state-dependent dividends where state probabilities were either risky or ambiguous. Each asset paid a fixed dividend depending on the color of the ball drawn from a 3-color Ellsberg urn in which the number of balls for the risky market is known but the number of balls for the two risky assets is unknown. Thus, payoffs from the risky and the ambiguous asset are not independent from each other. Further, the composition of the balls in the urn changed over time as the balls were randomly drawn without replacement. The authors acknowledge that the reduction in ambiguity could be confounded with convergence to equilibrium, "because it is difficult - if not impossible - to assess when prices have "settled down" during an experimental period" (p.1351). Moreover, their setup required the computation of state price/probability ratios with a number of assumptions on priors and updating 7 Bossaerts et al. conclude that ambiguity aversion matters only partially in markets: "The predictions for portfolio choices seem quite robust and well supported by the experimental data; the predictions for prices are less robust" (p.1355).

Kocher and Trautmann (2013) designed two separate first-price sealed bid market environments for risky and ambiguous assets and allowed subjects to selfselect into one of two mutually exclusive markets. Hence, arbitrage between risk and ambiguity was not possible. Although less subjects chose to submit a bid in the ambiguous market, Kocher and Trautmann (2013) found no differences in the average transaction prices of risky and ambiguous assets.

In a recent study, Corgnet et al. (2013) modeled and experimentally tested a market with ambiguity averse traders. In their model, the market is divided into three categories: a proportion of rational traders who are ambiguity neutral; a proportion of noise traders who buy or sell the asset independent of the fundamentals; and a proportion of ambiguity-averse traders who maximize the

\footnotetext{
${ }^{6}$ The authors acknowledge that this is unexpected, as simultaneous auctions allow for arbitrage: "It seems that a more transparent comparison between the unambiguous and ambiguous assets leads to a greater differential in market prices (simultaneous versus independent) contrary to our expectation" (Sarin and Weber 1993 p.612).

${ }^{7}$ As there exists no theory for updating under ambiguity, the authors "follow the simplest approach and use uniform priors over the ambiguous states for the initial draw, updated by Bayes' Rule for subsequent draws" (p.1347). Although recent evidence points in the direction of Bayesian learning under ambiguity, it also suggests that subjects might over-adjust/underadjust to contradictory/confirming signals Qiu and Weitzel 2013).
} 
minimum expected utility over a set of multiple priors according to the maxmin model of Gilboa and Schmeidler (1989) $]^{8}$ Corgnet et al. (2013) showed theoretically that if the proportion of ambiguity-averse traders is greater than zero, the expected price of an ambiguous asset (with either a high or low dividend with unknown probabilities) is strictly lower than in a comparable baseline treatment with a risky asset. From the model, they predicted increased volatility, reduced trading volumes, and a greater dispersion of final asset holdings under ambiguity. Corgnet et al. (2013) experimentally tested their predictions in separate double auction markets in which a unique asset, either risky or ambiguous, was traded. Hence, arbitrage between risk and ambiguity was not possible. The authors did not find any statistically significant evidence for ambiguity premiums or for ambiguity effects on price volatility, trading volume or final share holdings.

As summed up at the bottom of table 1 , none of the studies provides broad and definite evidence of the ambiguity effects in experimental asset markets. In fact, the largest study to date, with 18 separate markets, finds no ambiguity effects (Corgnet et al. 2013); in line with Camerer and Kunreuther (1989) and Kocher and Trautmann (2013) ? $^{9}$

\subsection{Development of hypotheses}

As explained in the introduction, we isolate and focus on condition (ii) of the EMH (independent deviations from rationality). Translated into our setting, condition (ii) requires countervailing effects of both ambiguity averse and ambiguity seeking market participants. In fact, following this requirement, it is not unlikely to find substantial heterogeneity in ambiguity attitudes in both directions: ambiguity aversion as well as ambiguity seekingness. Many studies provide evidence of ambiguity seekingness, especially, but not exclusively, if the likelihood of winning is low (e.g., Einhorn and Hogarth, 1985, Curley and Yates, 1989, Kahn and Sarin, 1988). Halevy (2007) reports a proportion of $35 \%$ of ambiguity-seeking individuals. Moreover, the distribution of ambiguity attitudes seems to be quite symmetric around neutrality. In their baseline condition Charness et al. (2013) find that $12 \%$ behave in accordance with ambiguity seekingness; $60 \%$ of the subjects behave consistent with expected utility; $20 \%$ choose randomly; and only $8 \%$ of the subjects exhibit ambiguity aversion. Corgnet et al.

\footnotetext{
${ }^{8}$ The model of Corgnet et al. $(2013)$ is a modification of Dow and da Costa Werlang (1992).

${ }^{9}$ For the sake of completeness: Chen et al. (2007) modeled and experimentally tested first and second price auctions in which the distribution of bidder valuations is unknown. They rejected ambiguity aversion in favor of ambiguity seekingness and concluded that ambiguity attitudes are context dependent. However, they consider two bidder 'markets' in which the bidders have different values for the asset while we consider markets with several traders and the same fundamental value for all participants.
} 
(2013) report that $33 \%$ of their subjects are ambiguity seeking, while $35 \%$ are ambiguity neutral, and $32 \%$ ambiguity averse. All these ambiguity attitudes are measured individually. Thus, the whole distribution of ambiguity attitudes is shifted toward aversion as ambiguity aversion is the typical finding for the average individual (Trautmann and Van De Kuilen, forthcoming). However, this should also apply to the average trader in a market 10 Hence, despite a high heterogeneity of ambiguity attitudes which in principal supports condition (ii), it is therefore very likely that the typical distribution of traders' attitudes in markets is biased toward ambiguity aversion, too. This violation of condition (ii) leads to our baseline hypothesis 1a on ambiguity premiums:

Hypothesis 1. (a) Market prices exhibit ambiguity premiums.

In its simplest form, hypothesis 1a refers to a biased representative agent (representing the average of individual expectations that are biased toward ambiguity aversion). The same hypothesis, however, can also be developed from models with heterogeneous agents, of which some are ambiguity neutral and some ambiguity averse (e.g., Corgnet et al., 2013, Bossaerts et al. 2010) 11

However, facing conflicting experimental evidence where ambiguity premiums are rarely found, we argue that there must be another factor that explains why the same population of (on average) ambiguity-averse individuals produces a higher average ambiguity premium in individual decision making than in markets. Heterogeneity in ambiguity attitudes that explicitly accounts for the interaction between ambiguity-seeking and ambiguity-averse traders in financial markets has not yet been modeled (Caskey, 2009, Easley and O'Hara, 2009, Ahn et al., 2011, Alonso and Prado, 2011, Corgnet et al., 2013, Guidolin and Rinaldi, 2013). We therefore build on recent experimental insights regarding ambiguity attitudes to develop two explanations, both of which are related to condition (ii).

The first explanation refers to the robustness of ambiguity attitudes, which do not seem to be very stable or consistent within the same person. In fact, Dürsch et al. (2013) find that about 30\% of their participants are inconsistent in their choices across two repetitions of exactly the same ambiguity task. Dimmock et al. (2012) report similar within-subject inconsistencies for $47 \%$ of their participants. In an extensive study Stahl (forthcoming) categorizes more than $60 \%$ as 'level-0' participants, who almost randomly make their decisions. Corgnet et al. (2013) investigate whether the measure of individual ambiguity

\footnotetext{
10 Bossaerts et al. (2010) report corresponding experimental evidence for heterogeneity in ambiguity attitudes in markets.

11 Corgnet et al. (2013) assume three trader types (ambiguity averse, neutral and noise traders) while Bossaerts et al. 2010 illustratively develop a similar hypothesis with two trader types (ambiguity-neutral trader, Type I, and ambiguity-averse trader, Type II).
} 
attitudes in individual decision-making tasks are able to explain individual trading behavior in markets. In line with the notion that the ambiguity attitudes of a large percentage of subjects are not very stable and almost random Corgnet et al. (2013) further find that the previously elicited ambiguity attitudes do not predict individual valuations, final holdings, or the number of completed transactions in markets with ambiguous assets.

The lack of robustness of individual ambiguity attitudes suggests that many subjects are susceptible to external influences that may shift or channel behavior under ambiguity into a specific direction. Trautmann and Van De Kuilen (forthcoming) refer to these influences as 'moderators of ambiguity attitudes.' One of these moderators is feedback from groups. Keck et al. (2011) find that decisions in (small) groups of three have a weak tendency toward ambiguity neutrality compared to individual decisions. Charness et al. (2013) study persuasion in mixed groups, which include all types of ambiguity attitudes. Interestingly, they find that ambiguity-neutral subjects are better able to persuade ambiguityseeking subjects and also ambiguity-averse subjects. By contrast, neither of the latter two attitudes seems to be more justifiable than the other. Charness et al. (2013) therefore conclude that ambiguity neutrality has a 'persuasive edge' over both ambiguity aversion and ambiguity seekingness.

Combining the insight that a large proportion of traders of ambiguous assets are inconsistent in their behavior and that ambiguity neutrality is the most persuasive attitude, we propose that the level of immediate market feedback can be an important moderator of traders' ambiguity attitudes. In an extension of Charness et al. (2013), we argue that the market feedback of ambiguity neutral traders, via prices as well as the order book, have a persuasive influence on other market participants, particularly if the non-neutral attitudes of the latter are unstable. In support of this notion, Banerjee and Kremer (2010) and Banerjee (2011) show theoretically and empirically that market participants with a divergence of opinion on the interpretation of public information use market prices to update their beliefs. In a related study, Hommes (2011) finds experimentally that participants are able to coordinate their individual forecasts of market prices "in the absence of any communication between subjects other than through the observed realized price, and without any knowledge of the predictions of other participants" (p.10).

To test the premise that market feedback acts as a moderator of ambiguity attitudes, we administer two different market institutions, which differ substantially in their level of feedback. On one hand we consider the continuous openbook double auction (DA) which provides intra-period information explicitly on prices, bids and asks, and implicitly on volume, volatility, and liquidity. On the other hand we consider the call market (CA) which by contrast provides only 
inter-period information on the clearing price. Accordingly, we expect the ambiguity effects to be stronger in CM than in DA. Hence, we state our hypothesis $1 b$.

Hypothesis 1. (b) In call markets ambiguity premiums are higher than in double auctions.

The second explanation builds on the fact that ambiguity aversion, as a population average, is often not very strong. Given the substantial heterogeneity of ambiguity attitudes it comes as no surprise that average ambiguity premiums vary greatly across different individual decision-making tasks, often being close to zero 12 As shown above, many distributions of ambiguity attitudes are only modestly biased in the direction of ambiguity aversion. It is prudent to assume that this also applies to markets ${ }^{13}$ However, as Miller (1977) pointed out, and in line with the divergence of opinion literature, investment decisions reflect the opinion of the optimistic traders and not the average evaluation of the population 14 Hence, if the distribution of ambiguity attitudes is very heterogeneous and only slightly biased toward ambiguity aversion, it is possible that the more optimistic subjects (ambiguity seekers) will determine the price ${ }^{15}$ We therefore argue that even if ambiguity aversion is, on average, reflected in the evaluation of assets in individual decision-making situations, ambiguity premiums can only survive in markets if the distribution of ambiguity attitudes is sufficiently biased toward ambiguity aversion.

This has two important implications for the design of our experimental market. First, we choose a setup that attempts to maximize ambiguity aversion. Subjects are able to simultaneously trade a risky and an ambiguous asset in two separated markets on a split screen. By displaying both markets on the same screen, we increase the comparability and salience of ambiguity, which has been shown to pronounce ambiguity aversion (Fox and Tversky, 1995; Chow and Sarin 2001, Fox and Weber, 2002, Qiu and Weitzel, 2011). Moreover, by separating the two markets with a dedicated trading account for each market, we eliminate the possibility for arbitrage between risk and ambiguity ${ }^{16}$ We use simple 2-color Ellsberg urns, as 3-color Ellsberg urns seem to lead to lower levels of ambiguity aversion (Trautmann and Van De Kuilen, forthcoming). We

\footnotetext{
${ }^{12}$ See table 1 in Trautmann and Van De Kuilen (forthcoming).

13 Corgnet et al. 2013 estimate that the effect of ambiguity on trading behavior is likely to be limited if less than $1 / 3$ of the population is ambiguity averse. Based on their model they show that a proportion of $32 \%$ ambiguity-averse traders (with $33 \%$ noise traders instead of ambiguity-seeking traders, and 35\% ambiguity-neutral traders) translates into an average ambiguity premium of at most $6 \%$.

${ }^{14}$ See Banerjee (2011) for a survey and the main effects of opinion divergence in markets.

15 Anderson et al. (2005) and Banerjee (2011), amongst others, provide empirical evidence that heterogeneous beliefs indeed matter for asset pricing.

${ }^{16}$ The elimination of arbitrage does not only increase the chances for ambiguity aversion, but is also needed to exclude condition (iii) of the EMH.
} 
produce the ambiguous asset with 'strong ambiguity' without the explicit specification of second-order probabilities ${ }^{17}$ This stands in contrast to studies that use a compound lottery for the ambiguous asset, which is often referred to as 'weak ambiguity' as the second-order probabilities are known (Camerer and Kunreuther, 1989 Sarin and Weber, 1993). Second, we introduce a treatment effect that has been reported to increase the magnitude of ambiguity aversion. Abdellaoui et al. (2011) show that ambiguity aversion is significantly higher for high probability events than for medium or low probability events. We replicated the urn design of Abdellaoui et al. 2011) and administered an objective winning probability of $50 \%$, which is used in most experiments with ambiguity, and of $75 \%$, in which subjects exhibit stronger preferences for risk and against ambiguity. If we should find ambiguity aversion in markets with a high winning probability, but not with a medium winning probability, we can infer support for the notion that a sufficiently high bias toward ambiguity aversion is needed in a distribution of heterogeneous ambiguity attitudes for ambiguity premiums to survive in markets. We therefore, in more general terms, state our hypothesis $1 c$.

Hypothesis 1. (c) For a high winning probability, ambiguity premiums are higher than for a medium winning probability.

We further predict that ambiguity leads to lower trading volume and increased volatility (Dow and da Costa Werlang, 1992, Epstein and Wang, 1994, Mukerji and Tallon, 2001, Routledge and Zin, 2009, Guidolin and Rinaldi, 2010; Illeditsch, 2011), and that the dispersion of final asset holdings will be larger under ambiguity as traders may overweight their portfolio with less ambiguous assets, as implied by models on the home bias and the familiarity bias (Uppal and Wang, 2003 Huang, 2007; Cao et al. 2011). Hence, analogue to hypothesis 1 we state hypotheses 2,3 , and 4 to complete the predictions on ambiguity effects.

Hypothesis 2. (a) Ambiguity decreases trading volume. (b) Ambiguity decreases trading volume in call markets more than in double auction markets. (c) Ambiguity decreases trading volume in markets with a high winning probability more than in markets with a medium winning probability.

Hypothesis 3. (a) Ambiguity increases price volatility. (b) Ambiguity increases price volatility in markets with a high winning proba-

\footnotetext{
${ }^{17}$ In a robustness check we degenerate the market setting into an individual auction against the computer by implementing a Becker-DeGroot-Marschak mechanism (Becker et al., 1964). We find significant ambiguity effects in this individual decision-making condition which shows that our operationalization of ambiguity is reliable.
} 
bility more than in markets with a medium winning probability 18 Hypothesis 4. (a) Ambiguity increases the dispersion in final asset holdings. (b) Ambiguity increases the dispersion in final asset holdings in call markets more than in double auction markets. (c) Ambiguity increases the dispersion in final asset holdings in markets with a high winning probability more than in markets with a medium winning probability.

Note that in all hypotheses part (a) is in line with Corgnet et al.(2013) reflecting a general result from the theoretical literature. We extend their hypothesis with respect to our conditions in part (b) and (c).

\section{Experimental Design}

We run a $2 \times 2 \times 2$ design with two market institutions (double auction referred to as DA and call market referred to as $\mathrm{CM}$ ), two objective winning probabilities ( $4 / 8$ referred to as 48 condition and $6 / 8$ referred to as 68 condition), and two asset types (risky asset $\mathrm{R}$ and ambiguous asset $\mathrm{A}$ ). As our outcome variables refer to the difference between the risky and ambiguous assets (ambiguity effect), the setup boils down to a classic $2 \times 2$ design with two market institutions and two winning probabilities. Table 2 provides an overview of the four experimental conditions as a comparison to table 1

Subjects traded risky assets and ambiguous assets, both of which we referred to as shares. The shares were simultaneously traded on a split screen with the risky market on the left-hand side (with light-blue background) and the ambiguous market on the right-hand side (with light-yellow background), or vice versa. ${ }^{19}$ The two markets on the split screen were administered separately, each with a separate account. Thus, arbitrage across markets was not possible. The treatments directly contrasted the risky with the ambiguous asset, which makes the ambiguous alternative more salient and pronounces ambiguity effects (Fox and Tversky, 1995). To avoid any reference to risk or ambiguity we referred to the shares on the two sides of the split screen as blue and yellow shares, respectively. At the beginning of each trading period, subjects were endowed with one of two endowment profiles. Four traders received ten blue shares and 4,200 ECU cash in the blue market and six yellow shares and 5,200 ECU cash in the yellow market. The other four traders received six blue shares and 5,200

\footnotetext{
${ }^{18}$ Note that CM treatments do not provide continuous trading data for intra-period volatility.

${ }^{19}$ We counter-balanced $\mathrm{R}$ and $\mathrm{A}$ on the left- and right-hand side of the split screen: four traders (randomly determined) always traded the risky asset on the left-hand side and the ambiguous asset on the right-hand side of the split screen, and the other four traders vice versa.
} 
ECU cash in the blue market and ten yellow shares and 4,200 ECU cash in the yellow market. In all endowments, 3,000 ECU of the cash portion was provided as a loan and had to be returned at the end of each period ${ }^{20}$ Thus, eight subjects belonged to one market cohort and the composition did not change throughout the entire experiment. Short sales were not allowed. Instructions along with all data at period level, screenshots and the laboratory set up can be found in the online supplements $\mathrm{C}, \mathrm{D}$ and $\mathrm{E}$

For each of the four conditions (CM48, CM68, DA48, DA68) we ran two sessions. Each experimental session consisted of 24 market participants organized into three cohorts and 12 consecutive and identically designed periods. Thus, our analysis is based on 24 cohorts with a total of 192 subjects. Our source of ambiguity is a non-computerized version of Abdellaoui et al. (2011, section III). All monetary values were denominated in experimental currency units (ECU) with an exchange rate of 200 ECU per Euro.

\subsection{Market Institutions}

In the DA conditions eight participants had the opportunity to buy and sell shares from each of the two markets in two standard computerized continuous open-book double auction markets. Each period lasted four minutes. The screen was organized in such a way that it simultaneously displayed the two independent double auction markets on the left- and right-hand side in the colors of the respective share.

In the CM conditions we replaced the double auction with a call market, keeping everything else equal. In each of the two markets, traders submitted a buy order by entering the maximum quantity of shares they were willing to buy and the highest price they were willing to pay. Furthermore, they submitted a sell order by entering the maximum quantity of shares they were willing to sell and the lowest price they were willing to accept. Then the computer matched the buy and sell orders to determine a market price for each of the two shares and then cleared each of the two markets separately. We used the programming code and implementation of Cheung and Palan (2012) ${ }^{21}$

\subsection{Risky Shares and Ambiguous Shares}

We followed the experimental design of Abdellaoui et al. (2011) and implemented a non-computerized version of their ambiguous urn. At the end of each period, each share paid either a low (124 ECU) or a high dividend (300 ECU). These dividend payments were determined by drawing a ball from an Ellsberg urn that contained eight balls with up to eight colors. If the drawn ball matched

\footnotetext{
${ }^{20}$ None of the subjects went bankrupt.

${ }^{21}$ We thank the authors for providing their zTree code.
} 
one of the $X$ winning colors, the corresponding share earned the high dividend; otherwise the share earned the low dividend. In condition 48 (four winning colors out of eight possible colors) the probability of drawing a winning color was $50 \%$. In condition 68 (six winning colors out of eight possible colors) we increased the objective winning probability to $75 \%, 22$

The $X$ winning colors were determined as follows. Upon arrival, and without any information about the experiment, each of the 24 subjects selected $X$ colors by independently and privately marking them on a sheet with the eight possible colors. Hence, each of the 24 sheets indicated $X$ privately chosen winning colors. The 24 sheets were pinned on the lab wall, organized in two columns such that each subject was able to see it. One column with 12 sheets represented the winning colors of the risky (= blue) shares for each of the 12 periods along with a blue colored poster named BLUE to identify the share. The other column with 12 sheets represented the winning colors of the ambiguous (= yellow) shares for each of the 12 periods along with a yellow colored poster named YELLOW ${ }^{23}$

For the risky share, we publicly filled one urn with exactly eight balls of eight different colors and placed it below the blue poster. After each period, a randomly chosen subject drew one ball from the risky urn and we compared the drawn color with the winning colors for that period to determine the dividend. To operationalize ambiguity, 12 different urns (one for each period) were created as follows. For each session we invited four additional subjects ('ambiguity providers'), who stayed in the waiting room outside of the lab. After the other 24 subjects were seated and marked their winning colors, we removed mobile blinds from a table with jars of enough balls to fill each urn with only one of the eight colors ${ }^{24}$ Then we asked the ambiguity providers to fill three urns each and concealed the table with the blinds again. Neither the experimenters nor the other subjects were able to see how the ambiguity providers filled the ambiguous urns (one by one) behind the blinds ${ }^{25}$ After the ambiguity providers handed over their ambiguous urns, we placed all 12 urns in a random sequence directly below the yellow poster in full view of all the subjects. Below the blue poster we placed the risky urn (after public disclosure of the contents of the risky urn). We then privately paid the ambiguity providers a fixed fee of six Euros

\footnotetext{
${ }^{22}$ Note that both urns are commonly referred to as '2-color Ellsberg urn' despite the fact that they contain up to eight colors and different winning probabilities. This is to differentiate it from '3-color Ellsberg urn problems' in which risky and ambiguous assets are mixed in one urn.

${ }^{23}$ A photo of the setup in the lab can be seen in the online supplement $\mathrm{C}$

${ }^{24}$ We provided $12 \times 8=96$ balls for each color in all other sessions, i.e., in total 768 balls.

${ }^{25}$ If the experimenter filled the urn decision makers may think that the experimenter had chosen a probability distribution with the objective of minimizing the subjects' expected utility (see discussion in Chow and Sarin $(2002)$ ). We avoid this situation by letting independent subjects fill the urn without any knowledge of the experiment.
} 
and dismissed them $\sqrt{26}$ After each period, a randomly determined subject drew a ball from one of the 12 ambiguous urns (randomly chosen by the subject) to determine the dividend of the ambiguous asset. The ambiguous urn was then removed. Comprehension questions revealed that subjects understood the dividend procedure for the risky share and for the ambiguous share precisely ${ }^{27}$

\subsection{Procedure and Payment}

All subjects were recruited by using ORSEE (Greiner, 2004). Subjects were invited from a broad student database across several fields of study. We only invited subjects, who had never participated in asset market experiments before ${ }^{28}$ All market sessions were run at the University of Düsseldorf (Germany). One session constituted 24 market participants, involving three cohorts of eight traders and four ambiguity providers. Apart from the end-of-period draw from the urns, the experiment was entirely computerized using z-Tree (Fischbacher, 2007). Dividends were entered using an experimenter screen. One session lasted approximately two hours. Subjects received an average payment of at least 20 Euros (see table 2).

The sequence of events was as follows. Upon arrival, market participants marked the winning colors in private and the ambiguity providers filled the ambiguous urns and were then dismissed. Market participants were then trained on-screen on how to use the computer interface. We explained step-by-step how to buy and sell assets. Then subjects took part in trial rounds to get accustomed to the interface. After the trial rounds, we carefully explained the actual experiment and in particular the determination of dividend payments 29 We then privately answered remaining questions and started the first of the 12 payoff-relevant periods. After period 12, we employed the random incentive system to determine payments (Starmer and Sudgen, 1991; Hey and Lee, 2005). One of the 12 periods was individually and randomly selected (with a 12-sided dice). Within the payoff-relevant period we selected one of the two markets of the split screen (blue or yellow) with a coin flip. Traders received the end-ofperiod net cash plus the dividends of their share holdings from that market plus

\footnotetext{
${ }^{26}$ The blinds stayed around the table with the jars throughout the whole experiment so that nobody (also not the experimenters) could see the remaining balls in the jars. To make sure that there were eight balls in each ambiguous urn, we counted the number of balls through the fabric of the urns (bags).

${ }^{27}$ In the 68 condition subjects had a short questionnaire to check whether they understood the procedure, and $96 \%$ of all students answered all questions correctly. As the difference between the 68 condition and the 48 condition is minimal, we are confident that the students in the 48 condition understood the procedure as well.

${ }^{28}$ To minimize the confounding effects and variance arising from gender compositions in experimental assert markets as shown in Eckel and Füllbrunn (2013), we only invited male students. Unfortunately, in CM48 two cohorts also include some female traders as not enough males registered for the experiment. As we find no indication for differences between pure male market and mixed markets, we do not report them separately.

${ }^{29}$ All instructions and z-Tree codes are available from the authors upon request.
} 
show-up fee ${ }^{30}$ Thereafter, they answered a short demographic questionnaire, were privately paid in cash and dismissed.

\section{Results}

We search for ambiguity effects by using the within period differences between the risky $(R)$ market and the ambiguous $(A)$ market as the relevant unit of observation. To test our hypotheses we consider the following measures:

1. ambiguity premium: price difference between the risky and the ambiguous shares $\left(A P=P_{R}-P_{A}\right){ }^{31}$

2. difference in trading quantity: $Q D=$ Quantity $_{R}-$ Quantity $_{A}$,

3. difference in volatility: difference in the standard deviation of prices (in DA only), i.e., $S t D e v D=S t D e v_{R}-S t D e v_{A}$, and

4. difference in dispersion (standard deviation) of final share holdings:

$$
\text { DisD }=\text { Dispersion }_{R}-\text { Dispersion }_{A} .
$$

To evaluate whether markets show ambiguity effects within conditions, we test the Null that the suggested measures are equal to zero (no ambiguity effect). We provide results on four test methods. First, we run a Wilcoxon signed-rank test on each period and each condition with six independent observations. Second, we run a Wilcoxon signed-rank test on the average of all 12 periods and each condition with six independent observations. Third, we consider a Wilcoxon signed-rank test in line with Haruvy and Noussair (2006) (henceforth HN test) treating the period average in each condition as the relevant unit of observation yielding 12 observations from 12 periods for each condition. Fourth, we allow for a higher number of observations by pooling the observations in two blocks separately. In block I we pool the first six periods and in block II we pool the last six periods. Thus, we have 36 observations from six cohorts in six periods in each block 32

To compare across conditions, we use the HN test on the period differences of average condition measures between conditions and the Mann-Whitney U

\footnotetext{
${ }^{30}$ The show-up fee was 4 Euro in the 48 condition markets and 3 Euro in the 68 condition markets.

${ }^{31}$ In (Trautmann and Van De Kuilen forthcoming) ambiguity premium is defined as the price difference between the risky and the ambiguous asset relative to either the risk-neutral price or the price of the risky asset. We define the ambiguity premium as the difference between prices to allow for a comparison across conditions as the other measures are biased due to the differences in winning probabilities or general price level effects across conditions. For robustness we reran all tests with an ambiguity premium relative to the expected value, but find no qualitative difference to the reported results based on our measure.

${ }^{32}$ The third and fourth test assume that average period condition measures are independent over periods, resulting in equal weights for all periods.
} 
tests within blocks. We use a $95 \%$ confidence interval as a standard level of statistical significance $(p<0.05)$ unless otherwise stated. In the following we provide tables with aggregate data. Detailed tables on period data and also on prices can be found in the online supplement $\mathrm{D}$. The tables provide further information on test statistics and measures.

\subsection{Ambiguity Premium}

For each period, we computed the difference of prices between the risky share and the ambiguous share, i.e., $A P=P_{R}-P_{A}$. In DA we consider median period prices and in $\mathrm{CM}$ we consider clearing prices. To evaluate whether markets show ambiguity effects on prices, we test the Null that $A P$ is equal to zero for each condition separately. Table 3 reports the condition averages for each period $(n=6)$ along with p-values according to the above-mentioned tests. A positive $A P$ indicates ambiguity aversion. The average $A P$ over all periods is 1.84 ECU in DA48 and 2.42 ECU in DA68. However, as the test statistics in table 3 suggest, we cannot reject the Null in either DA condition (apart from two periods). The average $A P$ over all periods is $3.42 \mathrm{ECU}$ in CM48 and 8.54 ECU in CM68. While we cannot reject the Null in CM48 we find ample support for a positive $A P$ in $\mathrm{CM} 68$. The average $A P$ in each period is positive, in seven out of 12 periods $A P$ is significantly positive at least at the $10 \%$ level, and for all other tests we find the differences to be significant ${ }^{33}$

To provide an intuition of whether the ambiguity premium is economically significant, we calculated the relative ambiguity premium using the expected value as a reference, i.e., $A P / 212$ in condition 48 and $A P / 256$ in condition $68{ }^{34}$ The overall relative ambiguity premium is $3.32 \%$ in CM68 while the premium is only $0.87 \%$ in DA $48{ }^{35}$ Figure 1 depicts the average relative ambiguity premium over time. While conditions DA48, DA68, and CM48 have values above and below zero, in CM68 we find that the relative ambiguity premium is positive and relatively constant over time.

Table 4 provides the difference on the average $A P$ for each comparison of conditions, and the test results on the Null that differences in $A P$ between

\footnotetext{
${ }^{33}$ Dividend payments might have an effect on pricing in subsequent periods due to e.g., the gambler's fallacy. Using a random effects regression model or an OLS model clustered on sessions we find no significant effects of the lagged dividends on ambiguity premium considering all observations (263) or each condition separately (with 66 observations each). The dependent variable was the price difference and independent variables were a dummy $0 / 1$ for the low $/$ high lagged dividend payment for the risky share, a dummy $0 / 1$ for the low/high lagged dividend payment for the ambiguous share and the interaction of both. Results are available from authors on request.

${ }^{34}$ In condition 48 the expected value is $124 \times 0.5+300 \times 0.5=212$ and in condition 68 the expected value is $124 \times 0.25+300 \times 0.75=256$.

${ }^{35}$ Note that the relative ambiguity premium seems quite small in comparison to Sarin and Weber 1993) which is above $66 \%$ in one of the double auction market treatments. This is due to the fact that our expected value is already quite high.
} 
conditions are equal to zero. First we take a separate look at the effect of the market institutions for each condition 48 and 68 . The average difference is 1.58 ECU in the 48 condition and $6.09 \mathrm{ECU}$ in the 68 condition, which suggests that the ambiguity premium is higher in CM than in DA. In the 48 condition, however, we cannot support a treatment effect of the market institution as none of the tests reject the Null. In the 68 condition, however, we find support for a higher ambiguity premium in $\mathrm{CM}$ than in $\mathrm{DA}$ as the $\mathrm{HN}$ test is significant at the $10 \%$ level and the Mann-Whitney $\mathrm{U}$ test is significant at the $1 \%$ level in block II. However, we find no significant differences in block I. Second, we investigate the probability effect for each institution separately. The average difference is $-5.09 \mathrm{ECU}$ in $\mathrm{CM}$ and $-0.58 \mathrm{ECU}$ in DA, which suggests that the ambiguity premium is higher in the 68 condition than in the 48 condition. In DA, we cannot support a probability effect as none of the tests rejects the Null. In $\mathrm{CM}$ we do find support for a higher ambiguity premium in the 68 condition compared to the 48 condition as the block I test is significant at the $10 \%$ level and the block II test is significant at the $1 \%$ level. However, using the $\mathrm{HN}$ test we do not find a statistically significant effect ${ }^{36}$

Summing up, we can state the following result 1 for the ambiguity effect on the ambiguity premium.

Result 1. (a) We find a significant ambiguity premium, but only in the call market with a high winning probability. (b) We find weak support for a higher ambiguity premium in the call market than in the double auction market, but in the high probability condition only. (c) We find weak support for a higher ambiguity premium in the high probability condition, but in call markets only.

\subsection{Volume and Volatility}

For each period, we computed the difference between the number of trades of the risky share and of the ambiguous share, i.e., $Q D=$ Quantity $_{R}-$ Quantity $_{A}$. Table 5 reports condition averages of $Q D$ for each period along with p-values from the Wilcoxon signed-rank tests. A positive (negative) $Q D$ indicates that more risky (ambiguous) shares have changed hand. For CM48, DA48, and DA68 we find no indication of an ambiguity effect on volume. However, in CM68 we find significant differences in each test though not in each period, which shows that the number of executed trades is significantly higher for the ambiguous

\footnotetext{
${ }^{36}$ As a robustness check, we run the same tests with the relative ambiguity premium. The results are qualitatively in line with table 4 We find no significant differences in DA. We find significant differences in CM in block II $(\mathrm{p}=0.0018)$ and marginally significant differences at the $10 \%$ level in block I $(p=0.0898)$. Using the HN test, we find no statistically significant difference.
} 
share. On average (all periods) the trading volume in ambiguous markets is $28 \%$ higher than in risky markets.

Table 6 reports the difference of the average $Q D$ for each comparison of conditions, and test results for the Null that differences in $Q D$ between conditions are equal to zero. First, we take a separate look at the effect of the market institution for each condition 48 and 68 . The differences of $Q D$ between conditions $\mathrm{CM}$ and $\mathrm{DA}$ are both negative, indicating that $Q D$ is higher in $\mathrm{CM}$ than in DA. But only in the 68 condition do we find support for an ambiguity effect as all three tests suggest significant differences at the $10 \%$ level. Second, we take a separate look at the probability effect for each institution. While we do not find a probability effect in DA, it quite clearly exists in CM as the block I test is significant at the $10 \%$ level and the HN test and the block II test are significant at and below the $5 \%$ level. The results indicate that in CM the $Q D$ is higher in the 68 condition than in the 48 condition.

As tables 5 and 6 suggest, we find no ambiguity effect on the volatility of prices $\left(S t D e v D=S t\right.$ Dev $_{R}-S t$ Dev $\left._{A}\right)$ neither within (DA48, DA68) nor across conditions (DA48 vs. DA68) ${ }^{37}$

Summarizing, we can formulate results 2 and 3 focusing on ambiguity effects on volume and volatility.

Result 2. (a) Significantly more trades are executed in the ambiguous market than in the risky market only in the call market with high winning probability. (b) We find support that the difference in executed trades is higher in the call market than in the double auction market, but in the high probability condition only. (c) We find support that the difference in executed trades is higher in the high probability condition than in the medium probability condition, but in call markets only.

Result 3. We find no support of an ambiguity effect on volatility (a) within or (b) between conditions.

\subsection{Share Holdings}

For each period, we computed the difference of the standard deviation of endof-period-share holdings (henceforth dispersion) between the risky share and the ambiguous share, i.e., DisD $=$ Dispersion $_{R}-$ Dispersion $_{A}$. Table 7 reports the condition averages of DisD for each period along with p-values from the Wilcoxon signed-rank tests. A positive (negative) DisD indicates that the

\footnotetext{
${ }^{37}$ For robustness, we run the same tests with the ratio of standard deviations, i.e, with $\left(S t D e v_{R} / S t D e v_{A}\right)$. None of the tests (Block I, Block II, HN) is statistically different at the $5 \%$ level, in line with the results on $S t D e v D$.
} 
distribution of the end of period shareholdings is more dispersed for the risky share than for the ambiguous share. In CM68 we find a significant ambiguity effect in all tests (though not for each individual period), which shows that the distribution of shares is more dispersed in the ambiguous market than in the risky market.

A comparison between the conditions yields some support for the ambiguity effect on dispersion to be lower in DA than in CM and to be lower in the 48 than in 68 condition (see table 8). However, the support is weak as significant differences can only be found at the $10 \%$ level for the HN test and at the $5 \%$ level for only one of the two blocks.

Summing up, we state result 4 on the effect of ambiguity on the dispersion of end-of-period-share holdings.

Result 4. (a) The dispersion of end-of-period-share holdings is higher for the ambiguous share than for the risky share only in the call market with a high winning probability. (b) We find weak support that the difference in dispersion is higher in the call market than in the double auction market, but in the high probability condition only. (c) We find weak support that the difference in dispersion is higher in the high probability condition than in the medium probability condition, but in call markets only.

\section{$5 \quad$ Discussion and Robustness}

In general our results show that ambiguity effects arise only when certain conditions are met. First, we only found ambiguity effects in markets without any intra-period feedback. Second, given such markets, we found the ambiguity effects to be most pronounced when ambiguity attitudes were sufficiently biased toward ambiguity aversion in the high-winning probability condition.

We start the discussion with our baseline hypothesis that ambiguity premia survive in markets (H1a). We find ambiguity premia in the high winning probability condition in call markets, but not in any of the double auction markets. The latter is in line with most of the previous studies which primarily employed double auctions and also did not find any statistically robust ambiguity premia (Camerer and Kunreuther, 1989 Bossaerts et al. 2010, Kocher and Trautmann, 2013 Corgnet et al., 2013). Sarin and Weber (1993), however, report ambiguity premiums in double auctions, even when they allowed for arbitrage between the risky and the ambiguous asset. The design of their experiments 7 and 14 share similarities to our DA48 condition, but in contrast to their study, we find no 
ambiguity premium in DA48 ${ }^{38}$ One reason might be the difference in the implementation of the double auction market. We used a computerized anonymous double auction design while Sarin and Weber (1993) used an open outcry double auction. In the latter, subjects are not anonymous and are under observation by peers. This peer-effect has been frequently found to increase ambiguity aversion (Curley et al., 1986, Trautmann et al. 2008, Muthukrishnan et al., 2009). An intuitive explanation for this may be that subjects try to avoid losses more when they are publicly observable, but it is still unclear what drives this effect (Trautmann and Van De Kuilen, forthcoming). Further, in Sarin and Weber (1993) the shares paid either zero or 100 ECU while in our setting the shares always paid a positive amount (as in Corgnet et al. (2013)). Reviewing the literature on ambiguity premiums Trautmann and Van De Kuilen (forthcoming) concluded that "positive (rather than zero) payoffs in case the bet is lost strongly reduce the premium." Although the differences in the minimum dividend might explain Sarin and Weber's (1993) results, Bossaerts et al. (2010) and Kocher and Trautmann (2013) also have minimum dividends of zero, but find no robust evidence for ambiguity premiums. Finally, Sarin and Weber (1993) allowed for short sales, which enable ambiguity-/risk-averse traders to price in their pessimistic beliefs, effectively leading to lower prices (Miller, 1977). Indeed, the only two studies that report ambiguity effects also both allowed for short sales (Sarin and Weber, 1993 Bossaerts et al. 2010). However, final shareholdings in Sarin and Weber (1993) suggest no effects from short sales ${ }^{39}$ It is therefore questionable whether short sales have driven their results (also see further below).

Our evidence for ambiguity premia in the high winning probability condition in call markets provides support for both hypothesis $1 \mathrm{~b}$ and $1 \mathrm{c}$, but primarily in combination. In accordance with hypothesis $1 \mathrm{~b}$ our results suggest that market feedback (or rather the lack of it) is a moderator of ambiguity as the ambiguity premium, particularly for high winning probabilities, is higher in call markets than in double auction markets. Building on evidence from group decision making, we argued that the 'persuasive edge' of the ambiguity neutral traders (Charness et al. 2013) has a stronger impact in markets with feedback than in markets without feedback. In markets with feedback the persuasive edge of ambiguity neutrality may be strengthened by an 'intrinsic taste to conformity in markets' (Goeree and Yariv, 2007), i.e., the adoption of the opinion

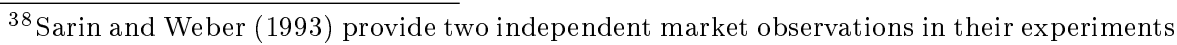
7 and 14. Our study provides six independent market observations for DA48 (and another six for DA68).

${ }^{39}$ Table 4 in Sarin and Weber (1993) shows that in experiment 7 and 14 (analogue to our DA48) the number of shares shorted is the same for the risky and the ambiguous asset. Bossaerts et al. 2010 provide no additional information on short sales.
} 
of other market participants on prices by neglecting own attitudes and beliefs. This might be especially prevalent in our experimental design, where subjects simultaneously trade in two markets with identical objective winning probabilities. Our split screen design was meant to increase ambiguity aversion, in line with prior evidence that ambiguity is less attractive when directly compared with risk (Fox and Tversky, 1995, Chow and Sarin, 2001, Fox and Weber, 2002, Qiu and Weitzel, 2011). This prior evidence, however, focused on one-shot individual decision making with no feedback. With continuous market feedback on both the risky and the ambiguous asset (with the same underlying winning probability), the split screen design may actually have the opposite effect with spillover effects of more 'intrinsic conformity' across both assets. Although the experimental evidence on spillover effects on prices in multiple asset markets is mixed (Palan, 2013), the split screen design could be a reason why we did not find any ambiguity premiums in markets with intra-period feedback (double auctions). To test for this possibility, we reran DA48, but with the difference that subjects traded either two risky shares or two ambiguous shares. Thus, each market of eight participants only traded one type of asset, mimicking the independent market setup of Sarin and Weber (1993) and Corgnet et al. (2013). The results of this robustness check showed no statistically significant difference in prices between the separately traded risky and ambiguous assets (see the online supplement $\mathrm{B}$ for detailed information on the design and the results). We are therefore confident that the split-screen design is not the reason for the missing ambiguity premiums in the double auction markets.

According to hypothesis $1 \mathrm{~b}$, market feedback within a trading period is the crucial mechanism, which is why we expect to see ambiguity effects in the CM, but not in the DA. This neglects the possibility of market feedback and learning across periods. Liu and Colman (2009) argue that ambiguity aversion is washed away with repetitions. Indeed, Sarin and Weber (1993) find weak convergence of prices over periods in some of their independent market settings. However, in reviewing the literature on ambiguity effects, Trautmann and Van De Kuilen (forthcoming) could not identify any consistent learning pattern, not even in individual decision making. In line with the latter study we also do not find learning patterns across periods. If inter-period market feedback plays a role, ambiguity effects should be weaker in later periods, in particular in the CM, where no other feedback is possible. Although we find an ambiguity premium right from the start in CM68, table 3 reports no tendency for it to disappear. In fact, even in the last three periods in CM68, there exists a significant ambiguity premium, suggesting a rather constant ambiguity effect. Also, neither of the other markets (CM48, DA48, DA68) display any consistent learning effects across periods (see table 3). 
In accordance with hypothesis $1 c$ we find evidence that only relatively strong biases toward ambiguity aversion survive in markets, but also only in call markets with limited feedback. Specifically, ambiguous assets with winning probabilities of $50 \%$ (or lower), as used in most previous studies (see table 1), do not seem to provide sufficiently strong ambiguity aversion. As Miller (1977) pointed out, investment decisions reflect the opinion of the optimistic traders. Our results support the notion that in markets with an only slightly biased population (DA48, CM48) there remains a sufficient number of optimistic subjects to prevent ambiguity premia. This requires, however, that our operationalization of ambiguity with the medium winning probability is sufficient to produce ambiguity effects even in individual decision making. If not, this could trivially explain why we do not find any effects in DA48 or in CM48. We therefore degenerated the two sided call market setting CM48 into an individual auction against the computer by implementing a Becker-DeGroot-Marschak mechanism (Becker et al. 1964). Subjects submitted their willingness-to-pay for one share in each of the two markets in 12 subsequent periods, using a split screen partitioning to stay close to the appearance of the market treatments. We refer to the online supplement $\mathrm{A}$ for information on the design and the results of this robustness check. We find that subjects' individual willingness-to-pay for the risky asset was significantly greater than for the ambiguous asset. However, although these results support our operationalization of ambiguity, alternative methods might have been even stronger. In our study we use a novel operationalization of ambiguity where external ambiguity providers filled the urns. The other studies mentioned in table 1 either used compound lotteries or did not tell subjects how the ambiguous urn was produced. If compound lotteries provided stronger ambiguity than our method, it could explain why Sarin and Weber (1993) found ambiguity premiums while we did not. As another robustness check we therefore ran the Becker-DeGroot-Marschak condition again, but this time produced the ambiguous asset with a compound lottery. The difference between the willingness-to-pay for the risky and the ambiguous assets turned out to be smaller and statistically significant only in one out of 12 periods. Hence, we are confident that our operationalization of ambiguity in the market experiments is sufficiently strong (and also stronger than compound lotteries used in Sarin and Weber (1993)).

Next to hypothesis 1 on the ambiguity premium we also found support for a higher dispersion of share holdings under ambiguity (H4). Again, the expected effects primarily hold for CM68, which confirms the importance of limited market feedback and a sufficient bias toward ambiguity aversion as important antecedents.

We found no support for our hypothesis 3 on price volatility. As there is 
no volatility measurement in call markets, $\mathrm{H} 3$ is the only hypothesis that solely focuses on double auctions. Analogous to hypothesis $1 \mathrm{~b}$ we barely find any ambiguity effects in the double auction market. The lack of support for $\mathrm{H} 3$ is therefore not very surprising. Moreover, it is in line with Corgnet et al. (2013), who also analyzed (and did not find) ambiguity effects on volatility.

Finally, our results do not support hypothesis 2 on the number of executed trades. In fact, we found statistically significant evidence of the exact opposite, namely that the number of executed trades increases under ambiguity. Our hypothesis 2 is based on Corgnet et al. (2013), who predict a lower number of trades under ambiguity. In a market consisting of noise traders, ambiguityneutral traders and ambiguity-averse traders, the latter have a price range (for long and short sales) in which they do not hold any ambiguous assets. Accordingly, the interaction with other traders in this specific price range is reduced, which leads to a lower number of trades in comparison to a setting without ambiguity-averse traders. As in our study, Corgnet et al. (2013) do not find support for this hypothesis. A reason for this may be that their model does not include market participants that are ambiguity seeking. Other models allow for ambiguity seekingness, but do not explicitly model the interplay between these opposite trader types 40 Our finding of a positive correlation between trading volume and ambiguity corresponds, however, with the divergence of opinions literature. Motivated by the optimistic pricing model of Miller (1977), this literature encompasses the whole spectrum of optimistic and pessimistic beliefs. It predicts not only a positive relation between belief dispersion and prices, but also a positive relation between dispersion and trading volume (Ajinkya et al. 1991). Many of these models, however, crucially rely on the presence of short sales constraints, which prevent the full revelation of information about pessimistic beliefs (Banerjee, 2011). Interestingly, all experiments that found indications of ambiguity aversion in markets did not have short sales constraints (Sarin and Weber, 1993 Bossaerts et al. 2010), while experiments that did not find any ambiguity effects had constraints on short sales (Camerer and Kunreuther, 1989 Corgnet et al. 2013). This suggests that short sales may be an additional condition for ambiguity effects to survive in markets. Although we did not allow for short sales in this study, we may have sufficiently cornered ambiguity effects in a specific market environment (CM68) so that short sales

\footnotetext{
${ }^{40}$ Equilibrium in such a setting is much more complicated. For example, Bossaerts et al. 2010 use the $\alpha$-maxmin model of Ghirardato et al. (2004) which is a generalization of the maxmin model by Gilboa and Schmeidler (1989) used in Dow and da Costa Werlang (1992); Corgnet et al. (2013). The $\alpha$-maxmin model allows for ambiguity seekingness if $\alpha<1 / 2$. However, to keep their model tractable, Bossaerts et al. (2010 p.1337) develop and illustrate their theoretical expectations with two types of agents: expected utility maximizers with a common prior (Type I), and ambiguity-averse agents with multiple priors and an $\alpha>1 / 2$ (Type II).
} 
were not necessary. The question, to which extent short sales support ambiguity effects in markets, is an interesting avenue for further research.

\section{Conclusion}

The lack of robust ambiguity effects in experimental asset markets suggests that subjects are susceptible to moderators of ambiguity. Using asset market experiments, we considered two potential moderators of ambiguity effects namely intra-period market feedback and the winning probability of the ambiguous asset in a $2 \times 2$ design. We implemented the first moderator by comparing a no-feedback-market, i.e., a call market, with a full-feedback-market, i.e., a double-auction market. We introduced the second moderator by increasing the winning probability for the ambiguous asset from $50 \%$ to $75 \%$. Such a shift has been shown to increase ambiguity aversion (Abdellaoui et al., 2011). In line with recent literature, we found no evidence for ambiguity effects in markets with a medium-winning probability. The same holds true in markets providing immediate feedback. However, we found clear ambiguity effects in markets without intra-period market feedback given sufficiently averse ambiguity attitudes toward assets with high winning probabilities.

In a recent study on ambiguity effects in markets, Corgnet et al. (2013) find no evidence for ambiguity effects and conclude "that the significance of the distinction between risk and ambiguity crucially hinges on the specific environment under study" (p.2). In this paper we were able to carve out conditions that need to be met for ambiguity effects to survive in specific market environments. A first essential condition is that the distribution of ambiguity attitudes should be sufficiently biased toward ambiguity aversion. The second condition is that feedback of other market participants needs to be limited. More theoretical and experimental research is needed to investigate other possible conditions and the market mechanisms under which ambiguity effects arise in markets.

\section{References}

Abdellaoui, M., Baillon, A., Placido, L., Wakker, P. P., 2011. The rich domain of uncertainty: Source functions and their experimental implementation. American Economic Review 101 (2), 695-723.

Ahn, D., Choi, S., Gale, D., Kariv, S., 2011. Estimating ambiguity aversion in a portfolio choice experiment. UC Berkeley Working Paper.

Ajinkya, B. B., Atiase, R. K., Gift, M. J., 1991. Volume of trading and the dispersion in financial analysts' earnings forecasts. Accounting Review, 389401. 
Alonso, I., Prado, J. M., 2011. Endogenous aggregate beliefs: Equity trading under heterogeneity in ambiguity aversion, working paper, Yale.

Anderson, E. W., Ghysels, E., Juergens, J. L., 2005. Do heterogeneous beliefs matter for asset pricing? Review of Financial Studies 18 (3), 875-924.

Banerjee, S., 2011. Learning from prices and the dispersion in beliefs. Review of Financial Studies 24 (9), 3025-3068.

Banerjee, S., Kremer, I., 2010. Disagreement and learning: Dynamic patterns of trade. The Journal of Finance 65 (4), 1269-1302.

Becker, G., DeGroot, M., Marschak, J., 1964. Measuring utility by a singleresponse sequential method. Behavioral Science 9 (3), 226-232.

Bossaerts, P., Ghirardato, P., Guarnaschelli, S., Zame, W. R., 2010. Ambiguity in asset markets: Theory and experiment. Review of Financial Studies 23 (4), 1325-1359.

Camerer, C., Kunreuther, H., 1989. Experimental markets for insurance. Journal of Risk and Uncertainty 2, 265-299.

Camerer, C., Weber, M., October 1992. Recent developments in modeling preferences: Uncertainty and ambiguity. Journal of Risk and Uncertainty 5 (4), 325-70.

Camerer, C. F., 1987. Do biases in probability judgment matter in markets? experimental evidence. The American Economic Review 77 (5), 981-997.

Cao, H. H., Han, B., Hirshleifer, D., Zhang, H. H., 2011. Fear of the unknown: Familiarity and economic decisions. Review of Finance 15 (1), 173-206.

Cao, H. H., Wang, T., Zhang, H. H., 2005. Model uncertainty, limited market participation, and asset prices. Review of Financial Studies 18 (4), 1219-1251.

Caskey, J. A., 2009. Information in equity markets with ambiguity-averse investors. Review of Financial Studies 22 (9), 3595-3627.

Charness, G., Karni, E., Levin, D., 2013. Ambiguity attitudes and social interactions: An experimental investigation. Journal of Risk and Uncertainty 46 (1), 1-25.

Chen, Y., Katuscak, P., Ozdenoren, E., 2007. Sealed bid auctions with ambiguity: Theory and experiments. Journal of Economic Theory 136 (1), 513-535. 
Cheung, S., Palan, S., 2012. Two heads are less bubbly than one: team decisionmaking in an experimental asset market. Experimental Economics 15, 373397.

Chow, C. C., Sarin, R. K., 2001. Comparative ignorance and the ellsberg paradox. Journal of risk and Uncertainty 22 (2), 129-139.

Chow, C. C., Sarin, R. K., 2002. Known, unknown, and unknowable uncertainties. Theory and Decision 52 (2), 127-138.

Corgnet, B., Kujal, P., Porter, D., 2013. Reaction to public information in markets: How much does ambiguity matter? The Economic Journal 123 (569), 699-737.

Curley, S. P., Yates, J., 1989. An empirical evaluation of descriptive models of ambiguity reactions in choice situations. Journal of Mathematical Psychology $33(4), 397-427$.

Curley, S. P., Yates, J. F., Abrams, R. A., 1986. Psychological sources of ambiguity avoidance. Organizational behavior and human decision processes 38 (2), $230-256$.

Dimmock, S. G., Kouwenberg, R., Wakker, P. P., 2012. Ambiguity attitudes in a large representative sample, working paper, Erasmus University Rotterdam.

Dow, J., da Costa Werlang, S. R., 1992. Uncertainty aversion, risk aversion, and the optimal choice of portfolio. Econometrica: Journal of the Econometric Society, 197-204.

Dürsch, P., Römer, D., Roth, B., 2013. Intertemporal stability of ambiguity preferences, working paper, University of Heidelberg.

Easley, D., O'Hara, M., 2009. Ambiguity and nonparticipation: the role of regulation. Review of Financial Studies 22 (5), 1817-1843.

Eckel, C., Füllbrunn, S., 2013. Thar 'she' blows? gender, competition, and bubbles in experimental asset markets, http://dx.doi.org/10.2139/ssrn.2271873.

Einhorn, H., Hogarth, R., 1985. Ambiguity and uncertainty in probabilistic inference. Psychological Review 92 (4), 433-461.

Ellsberg, D., 1961. Risk, ambiguity, and the savage axioms. The Quarterly Journal of Economics, 643-669.

Epstein, L. G., Noor, J., Sandroni, A., 2010. Non-bayesian learning. The B.E. Journal of Theoretical Economics 10 (1), 3. 
Epstein, L. G., Schneider, M., 02 2008. Ambiguity, information quality, and asset pricing. Journal of Finance 63 (1), 197-228.

Epstein, L. G., Wang, T., Mar. 1994. Intertemporal asset pricing under knightian uncertainty. Econometrica 62 (2), 283-322.

Etner, J., Jeleva, M., Tallon, J.-M., 2012. Decision theory under ambiguity. Journal of Economic Surveys 26 (2), 234-270.

Fama, E. F., 1970. Efficient capital markets: A review of theory and empirical work. The Journal of Finance 25 (2), 383-417.

Fischbacher, U., 2007. z-tree: Zurich toolbox for ready-made economic experiments. Experimental Economics 10 (2), 171-178.

Fox, C. R., Tversky, A., 1995. Ambiguity aversion and comparative ignorance. The Quarterly Journal of Economics 110 (3), 585-603.

Fox, C. R., Weber, M., 2002. Ambiguity aversion, comparative ignorance, and decision context. Organizational Behavior and Human Decision Processes 88 (1), 476-498.

Ghirardato, P., Maccheroni, F., Marinacci, M., October 2004. Differentiating ambiguity and ambiguity attitude. Journal of Economic Theory 118 (2), 133173.

Gilboa, I., Schmeidler, D., April 1989. Maxmin expected utility with non-unique prior. Journal of Mathematical Economics 18 (2), 141-153.

Goeree, J. K., Yariv, L., 2007. Conformity in the lab, working paper, Caltec.

Greiner, B., 2004. An Online Recruiting System for Economic Experiments. In: Kremer, K., Macho, V. (eds.), Forschung und wissenschaftliches Rechnen 2003. GWDG Bericht 63, Goettingen, Gesellschaft für wissenschaftliche Datenverarbeitung: 79-93.

Guidolin, M., Rinaldi, F., 2010. A simple model of trading and pricing risky assets under ambiguity: any lessons for policy-makers? Applied Financial Economics 20 (1-2), 105-135.

Guidolin, M., Rinaldi, F., 2013. Ambiguity in asset pricing and portfolio choice: a review of the literature. Theory and Decision 74 (2), 183-217.

Halevy, Y., 2007. Ellsberg revisited: an experimental study. Econometrica $75(2), 503-536$. 
Haruvy, E., Noussair, C. N., 2006. The effect of short selling on bubbles and crashes in experimental spot asset markets. The Journal of Finance 61 (3), 1119-1157.

Hey, J. D., Lee, J., 2005. Do subjects separate (or are they sophisticated)? Experimental Economics 8, 233-265.

Hommes, C., 2011. The heterogeneous expectations hypothesis: Some evidence from the lab. Journal of Economic Dynamics and Control 35 (1), 1-24.

Huang, R. R., 2007. Distance and trade: Disentangling unfamiliarity effects and transport cost effects. European Economic Review 51 (1), 161 - 181.

Illeditsch, P. K., December 2011. Ambiguous information, portfolio inertia, and excess volatility. Journal of Finance 66 (6), 2213-2247.

Kahn, B. E., Sarin, R. K., Sep. 1988. Modeling ambiguity in decisions under uncertainty. Journal of Consumer Research 15 (2), 265-272.

Keck, S., Diecidue, E., Budescu, D., 2011. Group decision making under ambiguity, working paper, Insead.

Keynes, J. M., 1921. A Treatise on Probability. London: MacMillan.

Klibanoff, P., Marinacci, M., Mukerji, S., 2005. A smooth model of decision making under ambiguity. Econometrica 73 (6), 1849-1892.

Kocher, M. G., Trautmann, S. T., 2013. Selection into auctions for risky and ambiguous prospects. Economic Inquiry 51 (1), 882-895.

Leippold, M., Trojani, F., Vanini, P., 2008. Learning and asset prices under ambiguous information. Review of Financial Studies 21 (6), 2565-2597.

Liu, H.-H., Colman, A. M., 2009. Ambiguity aversion in the long run: Repeated decisions under risk and uncertainty. Journal of Economic Psychology 30 (3), 277-284.

Maenhout, P. J., 2004. Robust portfolio rules and asset pricing. Review of Financial Studies 17 (4), 951-983.

Miller, E. M., 1977. Risk, uncertainty, and divergence of opinion. The Journal of Finance 32 (4), 1151-1168.

Mukerji, S., Tallon, J.-M., 2001. Ambiguity aversion and incompleteness of financial markets. The Review of Economic Studies 68 (4), 883-904.

Muthukrishnan, A., Wathieu, L., Xu, A. J., 2009. Ambiguity aversion and the preference for established brands. Management Science 55 (12), 1933-1941. 
Nau, R. F., 2006. Uncertainty aversion with second-order utilities and probabilities. Management Science 52 (1), 136-145.

Palan, S., 2013. A review of bubbles and crashes in experimental asset markets. Journal of Economic Surveys 27 (3), 570-588.

Qiu, J., Weitzel, U., 2011. Reference dependence and loss aversion in probabilities: Theory and experiment of ambiguity attitudes, http://dx.doi.org/10.2139/ssrn.1972293.

Qiu, J., Weitzel, U., 2013. Experimental evidence on valuation and learning with multiple priors, http://dx.doi.org/10.2139/ssrn.2207413.

Routledge, B., Zin, S., October 2009. Model uncertainty and liquidity. Review of Economic Dynamics 12 (4), 543-566.

Sarin, R. K., Weber, M., 1993. Effects of ambiguity in market experiments. Management Science 39 (5), 602-615.

Savage, L. J., 1954. The Foundations of Statistics. New York: Wiley.

Shleifer, A., 2003. Inefficient markets: An introduction to behavioral finance. Oxford university press.

Stahl, D. O., forthcoming. Heterogeneity of ambiguity preferences. Review of Economics and Statistics.

Starmer, C., Sudgen, R., 1991. Does the random-lottery incentive system elicit true preferences? an experimental investigation. American Economic Review 81, 971-978.

Trautmann, S., Van De Kuilen, G., forthcoming. Ambiguity attitudes. In: Keren, G., Wu, G. (Eds.), Blackwell Handbook of Judgment and Decision Making. Blackwell.

Trautmann, S., Vieider, F., Wakker, P., 2008. Causes of ambiguity aversion: Known versus unknown preferences 36 (3), 225-243.

Uppal, R., Wang, T., 2003. Model misspecification and underdiversification. The Journal of Finance 58 (6), 2465-2486.

Wakker, P., 2010. Prospect Theory: for Risk and Ambiguity. Cambridge University Press, Cambridge, UK. 


\section{Tables and Figures}

Table 1: Ambiguity in Asset Market Experiments

The table provides an overview of asset market experiments with ambiguity. Ambiguity refers to the operationalization of the ambiguous urn with either a compound lottery (weak ambiguity) or with an unknown distribution (strong ambiguity). Urn type refers either to a purely ambiguous asset (2-color) or a mix of ambiguous and risky assets in one urn (3-color). Dividends show the low and high dividend payments in ECU. Arbitrage refers to conditions which either allowed for arbitrage by trading risky and ambiguous assets simultaneously or which did not allow for arbitrage as assets were traded in independent markets. Domain refers to the gain or loss domain of the asset's lottery. Winning probability is the objective probability of earning the high 'dividend' payment (which is zero in Camerer and Kunreuther 1989). Market institutions were either the double auction (DA), the first price sealed bid auction (FPA), or the uniform price auction (UPA). The table further provides information on the number of independent markets (\# ind. obs.), the number of traders in a market, and the number of repetitions. In the results section the table reports statistically significant effects on prices, price volatility, number of executed trades, and the standard deviations of end of period stock holdings (Dispersion). For "-" the papers provide insufficient information to make a statement on the respective effect.

\begin{tabular}{|c|c|c|c|c|c|}
\hline & $\begin{array}{c}\text { Camerer/ } \\
\text { Kunreuther } \\
\text { (1989) }\end{array}$ & $\begin{array}{l}\text { Sarin/ } \\
\text { Weber } \\
(1993)\end{array}$ & $\begin{array}{l}\text { Bossaerts } \\
\text { et al. } \\
(2010)\end{array}$ & $\begin{array}{c}\text { Kocher/ } \\
\text { Trautmann } \\
\text { (2013) }\end{array}$ & $\begin{array}{l}\text { Corgnet } \\
\text { et al. } \\
(2013)\end{array}$ \\
\hline Country & US & GER & US & NL & US \\
\hline Ambiguity & weak & weak & strong & strong & strong \\
\hline Urn type & 2-color & 2-color & $3-\operatorname{color} a$ & 2-color & 2-color \\
\hline Dividends & $-20 /-40 /-100,0$ & 0,100 & 0,1 & 0,30 & $100,200^{b}$ \\
\hline Arbitrage & yes & yes $/$ no & yes & no & no \\
\hline Domain & loss & gain & gain & gain & gain \\
\hline Win. Prob. & $0.5-0.99$ & $0.05 / 0.5$ & $0.33^{c}$ & 0.50 & 0.50 \\
\hline Market & DA & $\mathrm{UPA} / \mathrm{DA}$ & DA & FPA & $\mathrm{DA}$ \\
\hline \# Ind. Obs. & 13 & $6^{d} \sqrt{4^{e}}$ & $6^{f}$ & 8 & $18^{g}$ \\
\hline \# Traders & $6-12^{h}$ & 8 & $24-29$ & $20-24$ & 8 \\
\hline \# Rep. & $10-35$ & $2-16$ & 8 & 1 & 2 \\
\hline \multicolumn{6}{|c|}{ Ambiguity effects found on } \\
\hline Price & No & $\mathrm{R}>\mathrm{A}^{i}$ & Not robust & No & No \\
\hline Volatility & - & - & - & - & No \\
\hline Volume & No & - & - & - & No \\
\hline Dispersion & - & No & $\mathrm{Yes}^{j}$ & - & No \\
\hline
\end{tabular}

\footnotetext{
${ }^{a} 3$-color urn with one market for each color.

${ }^{b}$ Expected dividends as dividends were drawn from either an urn containing equally likely dividends of 50,100, and 150 or an urn containing equally likely dividends of 100, 200, and

${ }^{c}$ Applies to beginning of trading only as balls were drawn without replacement.

${ }^{d}$ In Aachen 1 and 2 subjects traded both assets in different periods.

${ }^{e}$ In Aachen 3 and 4 subjects traded both assets in different periods.

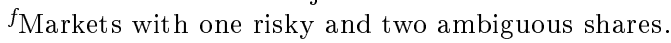

${ }^{g} 9$ ambiguous markets and 9 risky markets.

$h$ Traders were able only to buy or only to sell.

${ }^{i}$ Only with winning probability 0.5 . No test statistics.

Bossaerts et al. (2010) primarily analyzed ambiguity effects on the portfolio composition of modal investors and less on the asset dispersion across investors.
} 300 . 
Table 2: Overview of the conditions and results

For each condition (CM48, CM68, DA48, DA68) the table reports the experimental setup in comparison to the literature mentioned in table 1. We administered strong ambiguity with an unknown probability distribution. We did not allow for arbitrage as subjects had one separate account for each share. Although our ambiguous urn contains a maximum of eight colors it is not a mix of ambiguous and risky assets (3-color Ellsberg problem), but resembles a purely ambiguous asset (commonly referred to as '2-color' Ellsberg problem). Winning probability is the objective probability of earning the higher of the two dividends in the gain domain. Market institutions were either the double auction (DA) or the call market (CM). The table further provides information on the number of independent markets (\# ind. obs.), the number of traders in a market, and the number of repetitions. In the results section the table reports statistically significant effects on prices, price volatility, number of executed trades, and the standard deviations of end of period stock holdings (Dispersion). For "-" the papers provide insufficient information to make a statement on the respective effect.

\begin{tabular}{|c|c|c|c|c|}
\hline Condition & CM48 & CM68 & DA48 & DA68 \\
\hline Country & \multicolumn{4}{|c|}{ Germany } \\
\hline Ambiguity & \multicolumn{4}{|c|}{ strong } \\
\hline Urn type & \multicolumn{4}{|c|}{ 2-color } \\
\hline Dividends & \multicolumn{4}{|c|}{124,300} \\
\hline Arbitrage & \multicolumn{4}{|c|}{ No } \\
\hline Domain & \multicolumn{4}{|c|}{ gain } \\
\hline Win. Prob. & 0.50 & 0.75 & 0.50 & 0.75 \\
\hline Market & \multicolumn{2}{|c|}{ Call Market } & \multicolumn{2}{|c|}{ Double Auction } \\
\hline \# Ind. Obs. & 6 & 6 & 6 & 6 \\
\hline \# Traders & 8 & 8 & 8 & 8 \\
\hline \# Rep. & 12 & 12 & 12 & 12 \\
\hline \multicolumn{5}{|c|}{ Statistically significant effects on... } \\
\hline Price & No & $\mathrm{R}>\mathrm{A}$ & No & No \\
\hline Volatility & - & - & No & No \\
\hline Volume & No & $\mathrm{R}<\mathrm{A}$ & No & No \\
\hline Dispersion & No & $\mathrm{R}<\mathrm{A}$ & No & No \\
\hline$\varnothing$ Pay (Euros) & 20.60 & 22.10 & 21.43 & 22.06 \\
\hline
\end{tabular}


Table 3: Within condition effects: Ambiguity Premium

For each condition (CM48, CM68, DA48, DA68) the table reports the average of the difference of prices between the risky share and the ambiguous share, i.e., $A P=P_{R}-P_{A}$ (six observations for each condition). For the DA we consider the median prices and for the $\mathrm{CM}$ we consider the clearing prices. For each condition we provide p-values of Wilcoxon signed-rank tests. The asterisks indicate p-values referring to a Wilcoxon signed-rank test of the Null that the differences in a certain period are equal to zero $\left({ }^{*} \mathrm{p}<0.10,{ }^{*} \mathrm{p}<0.05\right)$. The $\mathrm{p}$-values in the row ' $\mathrm{p}$-value HN' refer to Wilcoxon signed-rank tests in line with Haruvy and Noussair (2006) of the Null that all condition averages of the 12 periods are equal to zero. The p-values in the row 'p-value block' refer to Wilcoxon signed-rank tests of the Null that all differences of the six cohorts in the first six periods (Block I) and in the last six periods (Block II) are equal to zero (36 observations for each condition and block). Data for each period can be found in the online supplement.

\begin{tabular}{c|cccc} 
Period & CM48 & CM68 & DA48 & DA68 \\
\hline 1 & 61.83 & $19.17^{*}$ & 20.50 & -8.50 \\
2 & $-19.00^{*}$ & 9.33 & 5.50 & -11.92 \\
3 & 2.67 & 2.33 & $9.00^{* *}$ & 2.25 \\
4 & -23.33 & $9.20^{*}$ & -0.58 & 12.08 \\
5 & 14.33 & $8.83^{*}$ & -5.33 & 5.67 \\
6 & -9.83 & 6.00 & 5.83 & 7.33 \\
7 & -5.00 & $10.67^{*}$ & -2.25 & 10.83 \\
8 & 10.83 & 7.67 & -1.00 & -0.92 \\
9 & -3.83 & 4.50 & 1.33 & 2.17 \\
10 & 4.50 & $6.67^{* *}$ & 5.25 & 2.67 \\
11 & 5.33 & $6.83^{*}$ & -7.75 & $7.75^{* *}$ \\
12 & 2.50 & $10.83^{* *}$ & -8.42 & -0.42 \\
\hline Average & 3.42 & $8.50^{* *}$ & 1.84 & 2.42 \\
\hline p-value HN & 0.814 & 0.002 & 0.583 & 0.209 \\
p-value block I & 0.931 & 0.001 & 0.092 & 0.208 \\
p-value block II & 0.573 & $<0.001$ & 0.597 & 0.114 \\
\hline
\end{tabular}


Table 4: Across condition effects: Ambiguity Premium

The table reports the differences of the average condition $A P(n=12 \times 6)$ between conditions. For example -5.09 is the difference between $A P$ in CM48 (3.42) and in CM68 (8.50) from table 3 . The p-values in the row 'p-value HN' refer to Wilcoxon signed-rank tests in line with Haruvy and Noussair 2006) of the Null that the period averages of conditions do not differ (12 observations). For example, for the first p-value 0.182 we compared row CM48 to row CM68 in table 3 . The p-values in the row 'p-value block' refer to the Mann-Whitney U tests of the Null that the rank sum of 36 differences are equal across conditions separately for block I and block II.

\begin{tabular}{ccccc} 
& 48 & 68 & $\mathrm{CM}$ & $\mathrm{DA}$ \\
Hypothesis & $\mathrm{CM}-\mathrm{DA}=0$ & $\mathrm{CM}-\mathrm{DA}=0$ & $48-68=0$ & $48-68=0$ \\
\hline Difference of Averages & 1.58 & 6.09 & -5.09 & -0.58 \\
\hline p-value HN & 1.000 & 0.060 & 0.182 & 0.583 \\
p-value block I & 0.397 & 0.224 & 0.062 & 0.991 \\
p-value block II & 0.567 & 0.004 & 0.001 & 0.135 \\
\hline
\end{tabular}


Table 5: Within condition effects: Volume and Volatility

For each condition (CM48, CM68, DA48, DA68) the table reports the average of the period differences between the number of trades in the risky market and in the ambiguous market, i.e., $Q D=$ Quantity $_{R}-$ Quantity $_{A}$ (six observations for each condition). For conditions DA48 and DA68 the table reports the average of the period differences between the standard deviation of prices in the risky market and in the ambiguous market, i.e., StDevD $=S t \operatorname{Dev}_{R}-S_{t} \operatorname{Dev}_{A}$ (six observations for each condition). For each condition we provide p-values of Wilcoxon signed-rank tests. The asterisks indicate p-values referring to a Wilcoxon signed-rank test of the Null that the differences in a certain period are equal to zero $\left(* \mathrm{p}<0.10,{ }^{* *} \mathrm{p}<0.05\right)$. The p-values in the row 'p-value HN' refer to Wilcoxon signed-rank tests in line with Haruvy and Noussair (2006) of the Null that all condition averages of the 12 periods are equal to zero. The p-values in the row 'p-value block' refer to Wilcoxon signed-rank tests of the Null that all differences of the six cohorts in the first six periods (Block I) and in the last six periods (Block II) are equal to zero (36 observations for each condition and block). Data for each period can be found in the online supplement.

\begin{tabular}{ccccccc} 
& \multicolumn{3}{c}{ Number of Trades } & \multicolumn{2}{c}{ Volatility } \\
\cline { 2 - 5 } Period & CM48 & CM68 & DA48 & DA68 & DA48 & DA68 \\
\hline 1 & 2.50 & -3.00 & -1.00 & 3.17 & 14.19 & -20.51 \\
2 & -1.67 & 1.17 & 2.00 & 4.33 & 27.88 & $27.04^{* *}$ \\
3 & 1.00 & $-8.33^{*}$ & 2.33 & 4.17 & $24.59^{* *}$ & 29.40 \\
4 & -3.17 & -3.33 & 2.17 & $-7.83^{* *}$ & -26.56 & -11.21 \\
5 & 0.67 & -1.83 & 3.83 & -6.50 & 1.50 & 22.19 \\
6 & -2.00 & -7.00 & $-7.50^{* *}$ & -0.50 & -55.92 & $-42.12^{*}$ \\
7 & -0.50 & -0.17 & 0.33 & 6.17 & 26.36 & 29.48 \\
8 & 2.17 & -6.50 & -1.17 & 1.33 & 75.16 & $9.50^{* *}$ \\
9 & -0.83 & -4.67 & -4.50 & -3.17 & -0.99 & 10.20 \\
10 & -1.00 & 1.17 & $-5.50^{* *}$ & -9.00 & 36.75 & $-28.72^{* *}$ \\
11 & 2.50 & $-4.17^{*}$ & 4.33 & 1.50 & -0.92 & 12.59 \\
12 & -1.67 & $-9.00^{*}$ & 4.33 & 4.33 & -27.21 & -12.48 \\
\hline Average & -0.17 & $-3.81^{*}$ & -0.03 & -0.17 & 7.90 & 2.11 \\
\hline p-value HN & 0.844 & 0.008 & 0.937 & 0.969 & 0.433 & 0.695 \\
p-value block I & 0.771 & 0.022 & 0.712 & 0.950 & 0.300 & 0.838 \\
p-value block II & 1.000 & 0.005 & 0.626 & 0.894 & 0.753 & 0.136 \\
\hline
\end{tabular}


Table 6: Across condition effects: Volume and Volatility

The table reports the differences of the average condition $Q D(n=12 \times 6)$ between conditions. For example 3.64 is the difference between $Q D$ in CM48 (-0.17) and in CM68 (-3.81) from table 5 The p-values in the row 'p-value HN' refer to Wilcoxon signed-rank tests in line with Haruvy and Noussair 2006) of the Null that the period averages of conditions do not differ (12 observations). For example, for the first p-value 0.182 we compared row CM48 to row CM68 in table 3 . The p-values in the row 'p-value block' refer to the Mann-Whitney U tests of the Null that the rank sum of 36 differences are equal across conditions separately for block I and block II.

\begin{tabular}{cccccc} 
& \multicolumn{4}{c}{ Volume } & Volatility \\
\cline { 2 - 5 } & 48 & 68 & $\mathrm{CM}$ & $\mathrm{DA}$ & DA \\
Hypothesis & CM-DA=0 & CM-DA $=0$ & $48-68=0$ & $48-68=0$ & $48-68=0$ \\
\hline Difference of Averages & -0.14 & -3.64 & 3.64 & 0.14 & 5.79 \\
\hline p-value HN & 1.000 & 0.084 & 0.023 & 0.844 & 0.695 \\
p-value block I & 0.870 & 0.094 & 0.093 & 0.937 & 0.753 \\
p-value block II & 0.584 & 0.094 & 0.008 & 0.866 & 0.184 \\
\hline
\end{tabular}


Table 7: Within condition effects: Dispersion of share holdings

For each condition (CM48, CM68, DA48, DA68) the table reports the average of the period differences between the standard deviation of end of period stock holdings in the risky market and in the ambiguous market, i.e., DisD $=$ Dispersion $_{R}-$ Dispersion $_{A}$ (six observations for each condition). For each condition we provide p-values of Wilcoxon signed-rank tests. The asterisks indicate p-values referring to a Wilcoxon signed-rank test of the Null that the differences in a certain period are equal to zero $\left({ }^{*} \mathrm{p}<0.10,{ }^{*} \mathrm{p}<0.05\right)$. The $\mathrm{p}$-values in the row 'p-value HN' refer to Wilcoxon signed-rank tests in line with Haruvy and Noussair 2006 of the Null that all condition averages of the 12 periods are equal to zero. The p-values in the row 'p-value block' refer to Wilcoxon signed-rank tests of the Null that all differences of the six cohorts in the first six periods (Block I) and in the last six periods (Block II) are equal to zero (36 observations for each condition and block). Data for each period can be found in the online supplement.

\begin{tabular}{c|cccc} 
Period & CM48 & CM68 & DA48 & DA68 \\
\hline 1 & 0.28 & -0.72 & -0.20 & $0.89^{* *}$ \\
2 & -1.43 & 0.55 & 0.21 & $-0.57^{*}$ \\
3 & 0.02 & -2.00 & -0.40 & 1.07 \\
4 & -0.94 & -1.51 & -0.52 & -0.04 \\
5 & 0.67 & -0.09 & $1.37^{*}$ & 0.62 \\
6 & -0.51 & -1.43 & 0.62 & 0.22 \\
7 & -0.62 & 0.07 & -0.51 & $1.42^{*}$ \\
8 & 0.23 & -1.63 & -0.34 & -0.68 \\
9 & -1.02 & $-1.43^{* *}$ & 0.02 & $-2.16^{* *}$ \\
10 & 0.12 & -0.85 & -0.35 & $-1.86^{* *}$ \\
11 & 0.52 & -0.52 & 0.16 & -0.54 \\
12 & -0.59 & $-1.93^{* *}$ & -0.10 & -0.31 \\
\hline Average & -0.27 & $-0.96^{* *}$ & 0.00 & -0.16 \\
\hline p-value HN & 0.272 & 0.008 & 0.583 & 0.754 \\
p-value block I & 0.338 & 0.081 & 0.566 & 0.144 \\
p-value block II & 0.489 & 0.001 & 0.753 & 0.046 \\
\hline
\end{tabular}


Table 8: Across condition effects: Dispersion of share holdings

The table reports the differences of the average condition DisD $(n=12 \times 6)$ between conditions. For example 0.69 is the difference between DisD in CM48 (-0.27) and in CM68 (-0.96) from table 7. The p-values in the row 'p-value HN' refer to Wilcoxon signed-rank tests in line with Haruvy and Noussair (2006) of the Null that the period averages of conditions do not differ (12 observations). For example, for the first p-value 0.182 we compared row CM48 to row CM68 in table 3. The p-values in the row 'p-value block' refer to Mann-Whitney U tests of the Null that the rank sum of 36 differences are equal across conditions separately for block I and block II.

\begin{tabular}{ccccc} 
Hypothesis & $\begin{array}{c}48 \\
\text { CM-DA }=0\end{array}$ & $\begin{array}{c}68 \\
\text { CM-DA }=0\end{array}$ & $\begin{array}{c}\mathrm{CM} \\
48-68=0\end{array}$ & $\begin{array}{c}\mathrm{DA} \\
48-68=0\end{array}$ \\
\hline Difference of Averages & -0.27 & -0.80 & 0.69 & 0.16 \\
\hline p-value HN & 0.272 & 0.060 & 0.050 & 0.583 \\
p-value block I & 0.300 & 0.029 & 0.386 & 0.581 \\
p-value block II & 0.893 & 0.362 & 0.022 & 0.188
\end{tabular}

Figure 1: Relative Ambiguity Premium

The figures depict the average relative ambiguity premium for the conditions DA48 and DA68 on the left-hand side and for the conditions CM48 and CM68 on the right-hand side. Each point represents the average of six observations from one period in a condition. The relative ambiguity premium is computed as the difference between risky and ambiguous prices $\left(P_{R}-\right.$ $P_{A}$ ) divided by the ambiguity neutral value which is 212 in the 48 condition and 256 in the 68 condition.
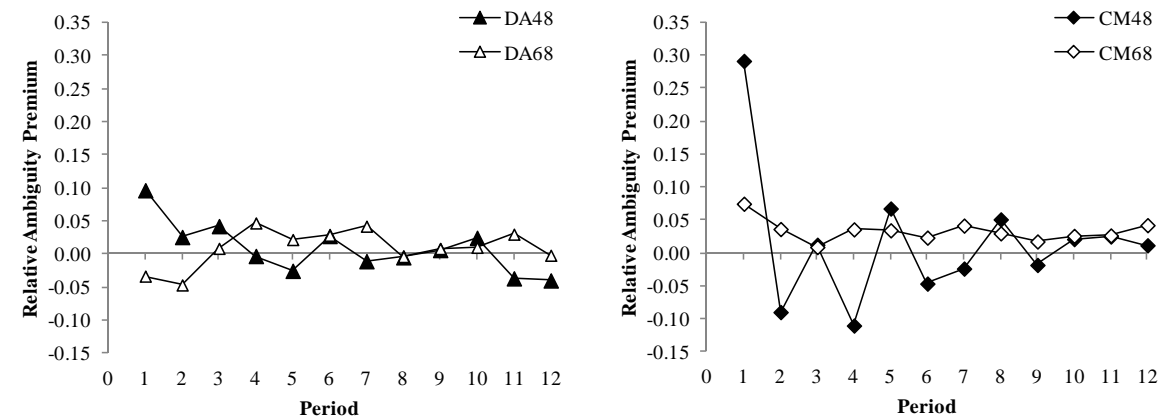


\section{Online Supplement}

\section{A Robustness Check: Individual Decision Mak- ing}

Given that we hardly find any ambiguity effects in 48 urn markets, it is important to test whether our operationalization of ambiguity is sufficient. Therefore, we elicited the individual willingness-to-pay (WTP) of subjects for the risky and the ambiguous share (48 urns) using a Becker-DeGroot-Marschak Mechanism (BDM). We again used the split-screen setup to stay close to the appearance of the other treatments (see Figure 2). We implemented the BDM mechanism as follows. Subjects submitted their WTP for one share in each of the two markets. The computer drew a random price for each market. If the subject's stated WTP exceeded the random price, the subject purchased the share at the random price. Otherwise, the subject did not purchase the share. As the WTP only applied to one share, we had to adjust the dividend by a factor of 10 (low/high dividend: 1,240/3,000 ECU) and the endowment to 3,500 ECU (including a 500 ECU loan). We operationalized ambiguity as in all DA and CM sessions by inviting ambiguity providers and letting subject choose their WTP in 12 subsequent repetitions.

We ran session BDM1 at the University of Düsseldorf with 20 participants who received an average payment of 16.55 Euro. We computed the individual bid differentials between share $R$ and $A$. Table 9 reports the average per period across all subjects. As expected, the average bids for $\mathrm{A}$ are lower in all periods and in the majority of periods this difference is also statistically significant. Moreover, the average differences across all periods and markets in Session BDM1 is also statistically significant. Applying the same test using all period differences from each subject in the first six periods (block I) and the last six periods (block II), we find differences to be highly significant indicating ambiguity aversion. The average relative ambiguity premium over all periods in $\mathrm{BDM}$ is $7.2 \%$. Although these results show that our operationalization works in individual decision making, it could still be possible that alternative operationalizations of ambiguity used in related studies are even stronger. The latter could explain why, for example, Sarin and Weber (1993) find ambiguity effects in markets while we do not.

To test this, we implemented a compound lottery in line with Sarin and Weber (1993) in a BDM2 session conducted at the NSM Decision Lab of Radboud University Nijmegen with 12 participants who receive an average payment of 16.58 Euro. We used the exact same source as we used in section III of 
Abdellaoui et al. (2011). This is basically the same procedure as in our other experiments except the fact that it is the computer which creates the ambiguous urn randomly instead of an ambiguity provider. Apart from the source of ambiguity, the experimental design is the same as in BDM1. Table 9 reports the average bid differentials between $\mathrm{R}$ and $\mathrm{A}$ for all subjects. Although the average bids for $\mathrm{A}$ are lower in most of the periods (with the exception of Period 12), only one of them is statistically significant. However, applying the same test and using all period differences from each subject in the first six periods (block I) and the last six periods (block II), we find the differences to be significant which indicates ambiguity aversion as well. The average relative ambiguity premium over all periods in BDM2 is $4.8 \%$ which is somewhat lower than in BDM1. Hence, overall, our primary operationalization of ambiguity by using additional subjects is not only sufficient to generate ambiguity effects in individual decision making, but seems to be stronger than alternative methods with compound lotteries used in related studies.

Figure 2: Screenshot of BDM split screen

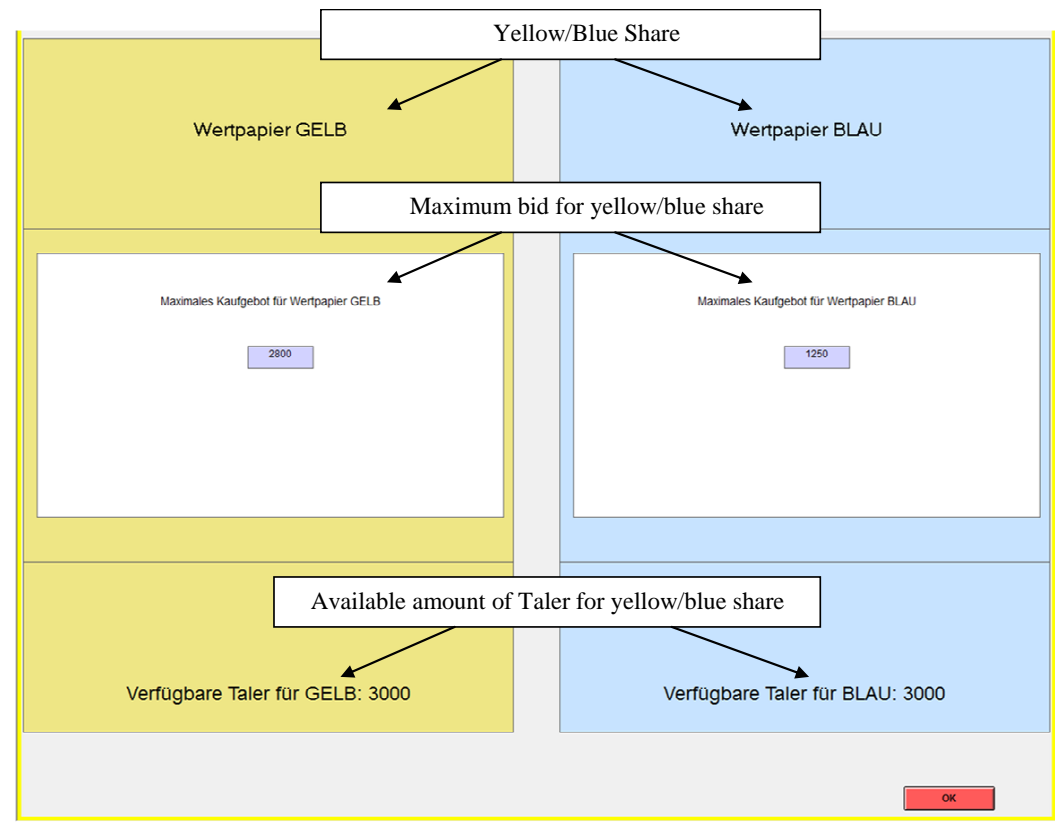


Table 9: Differences between $\mathrm{R}$ and A: bids in BDM

The table reports the average of differences between bids (willingness-to-pay) for the risky share and for the ambiguous share for BDM1, our primary operationalization of ambiguity, and for BDM 2 with ambiguity as a compound lottery (with known second-order probability). For each condition we provide $\mathrm{p}$-values of Wilcoxon signed-rank tests. The asterisks indicate p-values referring to a Wilcoxon signed-rank test of the Null that the differences in a certain period are equal to zero (* $\left.\mathrm{p}<0.10,{ }^{*} \mathrm{p}<0.05\right)$. The $\mathrm{p}$-values in the row ' $\mathrm{p}$-value HN' refer to Wilcoxon signed-rank tests in line with Haruvy and Noussair (2006) of the Null that all condition averages of the 12 periods are equal to zero. The p-values in the row 'p-value block' refer to Wilcoxon signed-rank tests of the Null that all differences of the six cohorts in the first six periods (block I) and in the last six periods (block II) are equal to zero ( 36 observations for each condition and block).

\begin{tabular}{c|cc} 
Period & BDM1 & BDM2 \\
\hline 1 & 97 & 114 \\
2 & 16 & 115 \\
3 & $176^{* *}$ & 10 \\
4 & 282 & 1 \\
5 & $265^{* * *}$ & 117 \\
6 & $174^{*}$ & 83 \\
7 & $242^{* * *}$ & 107 \\
8 & 11 & 142 \\
9 & $164^{* * *}$ & $202^{* *}$ \\
10 & 31 & 152 \\
11 & $171^{* * *}$ & 156 \\
12 & $29^{*}$ & -14 \\
\hline Average & $138^{* *}$ & $99^{*}$ \\
\hline p-value HN & 0.002 & 0.005 \\
p-value block I & $<0.001$ & $<0.001$ \\
p-value block II & 0.024 & 0.002 \\
\hline & &
\end{tabular}

\section{B Robustness Check: DA with only risky or only ambiguous shares}

In these sessions subjects traded either two risky shares (DA48R) or two ambiguous shares (DA48A). In each treatment we named one share the blue share and the other share the yellow share. The experimental design follows DA48 exactly, apart from the fact that in DA48R we made a random draw from the same urn with eight marbles and eight different colors and that in DA48A we had six ambiguity providers who provided 24 ambiguous urns. We applied the same procedure as in section 3.3. We conducted one DA48R session with three separated cohorts of eight traders who received on average 21.58 Euro and one DA48A session with three separated cohorts of 8 traders who received on aver- 
age 19.42 Euro (both at the University of Düsseldorf). Table 10 reports median prices along with p-values from two-sided Mann-Whitney U tests comparing prices between treatments and the HN test (see 4 for a description). To increase the number of observations in the tests, we pooled period prices in block I (periods 1 to 6 ) and block II (periods 7 to 12). The results show no indication for an ambiguity premium, i.e., that prices for the risky share exceeds prices for the ambiguous share. This result is line with Corgnet et al. (2013) who also considered independent markets and find no ambiguity aversion effect on prices.

Table 10: Ambiguity premium in DA48R and DA48A

The table reports median period prices and the 12 period average for the risky share in DA48R and for the ambiguous share in DA48A, separated by the color of the share (blue or yellow). The pvalues in the row 'p-value HN' refer to Wilcoxon signed-rank tests in line with Haruvy and Noussair (2006) of the Null that the differences of price averages of the 12 periods are equal to zero. The p-values in the row 'p-value block' refer to Mann-Whitney U tests of the Null that median prices in the first six periods (block I) and in the last six periods (block II) are equal to zero. Separating the tests by shares, we have 18 observations in each block. Also pooling also the shares, we have 36 observations in each block.

\begin{tabular}{|c|c|c|c|c|c|c|c|c|c|c|c|c|}
\hline \multirow{3}{*}{$\begin{array}{l}\text { Session } \\
\text { Cohort }\end{array}$} & \multicolumn{6}{|c|}{ Blue Share } & \multicolumn{6}{|c|}{ Yellow Share } \\
\hline & \multicolumn{3}{|c|}{ Risk } & \multicolumn{3}{|c|}{ Ambiguous } & \multicolumn{3}{|c|}{ Risk } & \multicolumn{3}{|c|}{ Ambiguous } \\
\hline & 1 & 2 & 3 & 1 & 2 & 3 & 1 & 2 & 3 & 1 & 2 & 3 \\
\hline 1 & 200 & 120 & 285 & 297 & 210 & 210 & 260 & 130 & 270 & 274 & 175 & 250 \\
\hline 2 & 205 & 100 & 245 & 244 & 215 & 225 & 200 & 109 & 240 & 214 & 210 & 220 \\
\hline 3 & 208 & 150 & 240 & 211 & 210 & 210 & 225 & 120 & 240 & 214 & 209 & 200 \\
\hline 4 & 200 & 138 & 235 & 250 & 205 & 180 & 220 & 140 & 235 & 250 & 210 & 160 \\
\hline 5 & 200 & 150 & 205 & 245 & 210 & 200 & 210 & 175 & 210 & 220 & 200 & 199 \\
\hline 6 & 220 & 180 & 230 & 180 & 209 & 180 & 220 & 180 & 202 & 180 & 208 & 181 \\
\hline 7 & 210 & 180 & 225 & 230 & 205 & 215 & 210 & 175 & 225 & 230 & 205 & 200 \\
\hline 8 & 220 & 170 & 235 & 220 & 210 & 220 & 215 & 170 & 230 & 245 & 210 & 191 \\
\hline 9 & 220 & 151 & 235 & 178 & 218 & 195 & 215 & 176 & 235 & 240 & 210 & 199 \\
\hline 10 & 225 & 190 & 235 & 235 & 215 & 200 & 210 & 188 & 225 & 222 & 210 & 195 \\
\hline 11 & 215 & 189 & 235 & 220 & 210 & 215 & 220 & 180 & 234 & 222 & 210 & 210 \\
\hline 12 & 220 & 185 & 235 & 190 & 210 & 215 & 221 & 180 & 235 & 229 & 210 & 215 \\
\hline Average & 212 & 159 & 237 & 225 & 211 & 205 & 219 & 160 & 232 & 228 & 206 & 202 \\
\hline \multicolumn{13}{|c|}{ Tests separated by share color } \\
\hline Diff. of averages & \multicolumn{6}{|c|}{-11} & \multicolumn{6}{|c|}{-8} \\
\hline p-value $\mathrm{HN}$ & \multicolumn{6}{|c|}{0.079} & \multicolumn{6}{|c|}{0.018} \\
\hline p-value block I & \multicolumn{6}{|c|}{0.124} & \multicolumn{6}{|c|}{0.924} \\
\hline p-value block II & \multicolumn{6}{|c|}{0.494} & \multicolumn{6}{|c|}{0.811} \\
\hline \multicolumn{13}{|c|}{ Tests both shares pooled } \\
\hline Diff. of averages & \multicolumn{12}{|c|}{-10} \\
\hline p-value HN & \multicolumn{12}{|c|}{0.029} \\
\hline p-value block I & \multicolumn{12}{|c|}{0.295} \\
\hline p-value block II & \multicolumn{12}{|c|}{0.817} \\
\hline
\end{tabular}




\section{Screen shots}

Figure 3: Screenshot of the split screen in the DA treatment

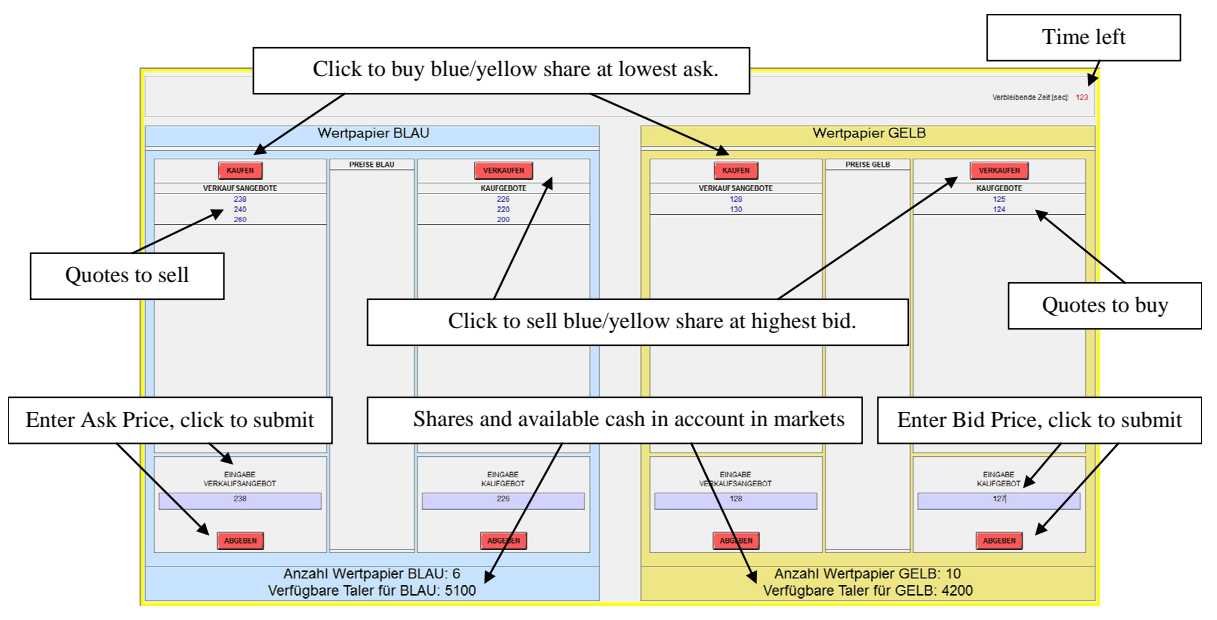

Figure 4: Screenshot of the split screen in the CM treatment

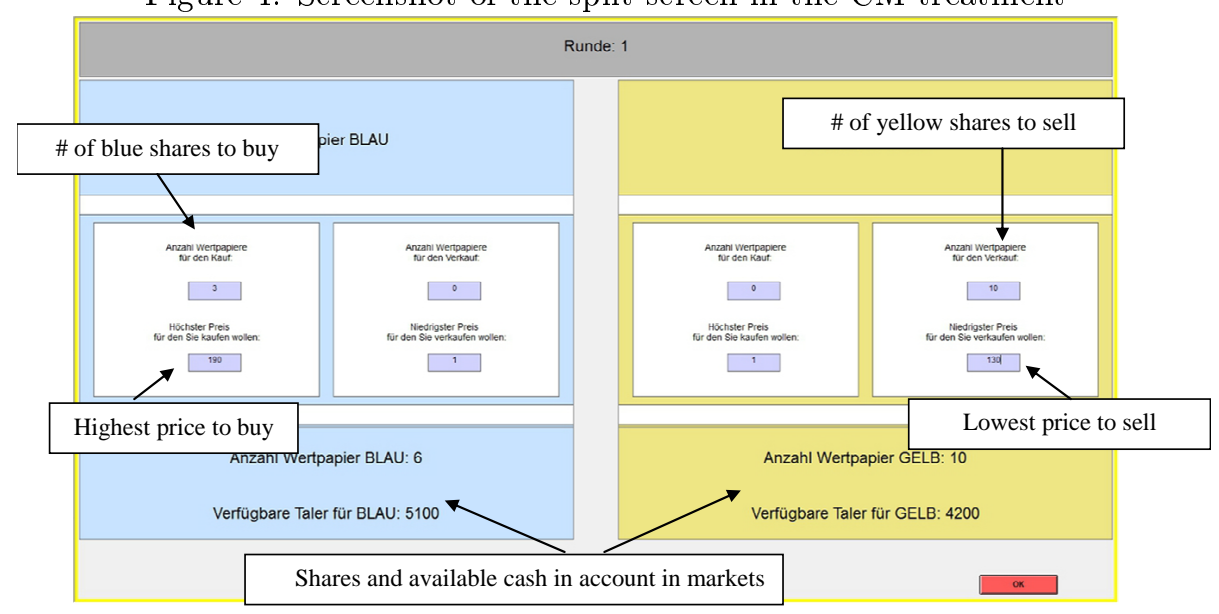


Figure 5: Setup in the Lab

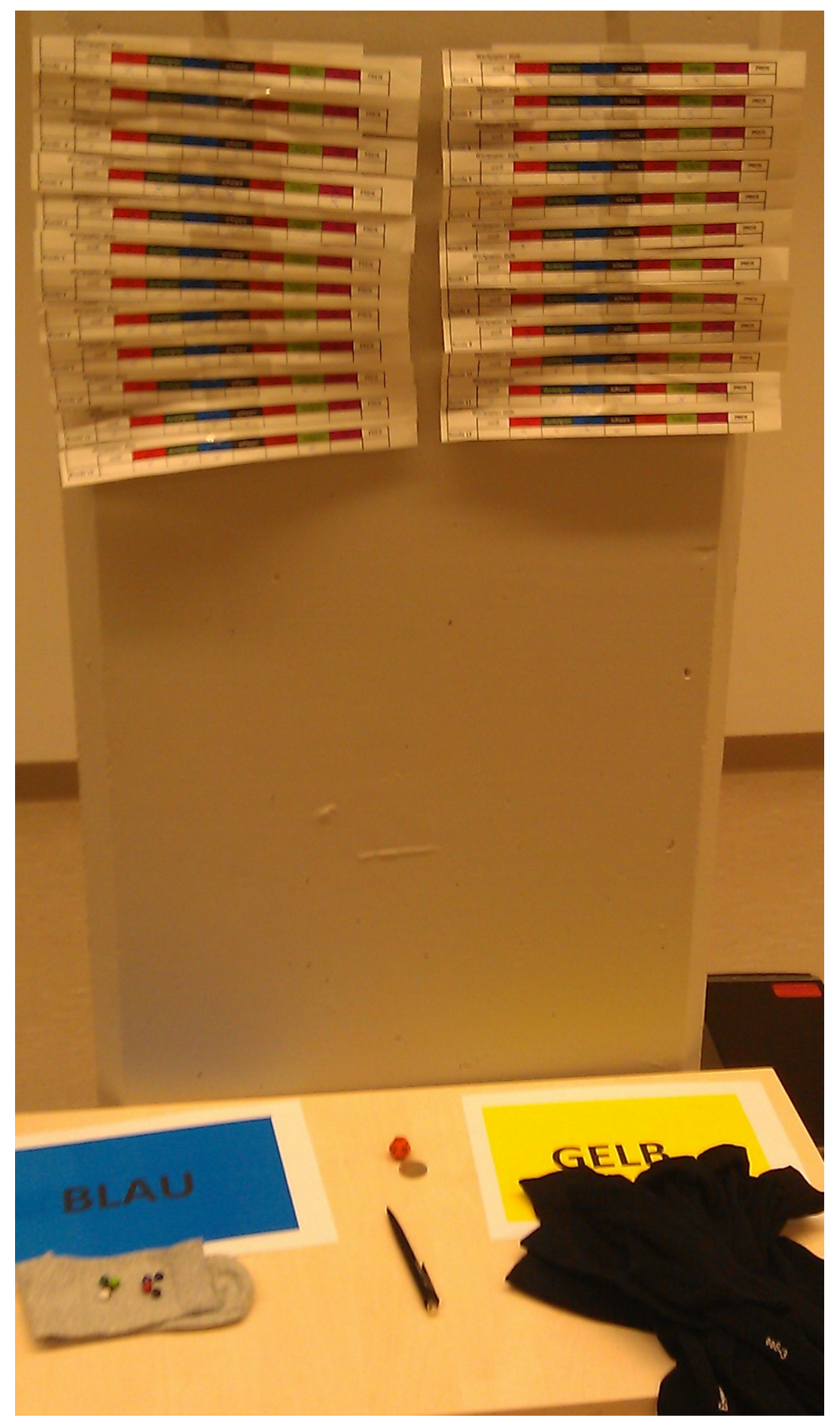




\section{Tables on period data}

Table 11: Periodical Data on Ambiguity Premium

For each condition (CM48, CM68, DA48, DA68) the table reports the price differences between the risky asset and the ambiguous asset. For the DA treatment we consider the median prices and for the $\mathrm{CM}$ treatment we consider the clearing prices.

\begin{tabular}{|c|c|c|c|c|c|c|c|c|c|c|c|c|}
\hline Period & 1 & 2 & 3 & 4 & 5 & 6 & 7 & 8 & 9 & 10 & 11 & 12 \\
\hline $\mathrm{CM} 48 / 1$ & -89 & -5 & -20 & 5 & 7 & -10 & -2 & 0 & 7 & -7 & 0 & 0 \\
\hline $\mathrm{CM} 48 / 2$ & 60 & -60 & -16 & -40 & -25 & -45 & -25 & 0 & -10 & 1 & 1 & 0 \\
\hline $\mathrm{CM} 48 / 3$ & 100 & -49 & 12 & 0 & 9 & 1 & 7 & 0 & 0 & 3 & 1 & 0 \\
\hline $\mathrm{CM} 48 / 4$ & 0 & 0 & 0 & -15 & -5 & 0 & 0 & 0 & 0 & 0 & 15 & 35 \\
\hline $\mathrm{CM} 48 / 5$ & 240 & 0 & 20 & -100 & 70 & -50 & 0 & -5 & -20 & 10 & -15 & 0 \\
\hline $\mathrm{CM} 48 / 6$ & 60 & 0 & 20 & 10 & 30 & 45 & -10 & 70 & 0 & 20 & 30 & -20 \\
\hline CM68/1 & 0 & 50 & 20 & . & 20 & 0 & 10 & 15 & 10 & 8 & -6 & 0 \\
\hline $\mathrm{CM} 68 / 2$ & 0 & 12 & 5 & 5 & 0 & 5 & 10 & 5 & 5 & 5 & 8 & 6 \\
\hline CM68/3 & 30 & 10 & -10 & 30 & 20 & 30 & 30 & 20 & 15 & 15 & 15 & 15 \\
\hline $\mathrm{CM} 68 / 4$ & 15 & 19 & 19 & 0 & 0 & -20 & 0 & 4 & 0 & 5 & 1 & 19 \\
\hline CM68/5 & 10 & 15 & 5 & 6 & 3 & 11 & 0 & -13 & 7 & 10 & 14 & 5 \\
\hline $\mathrm{CM} 68 / 6$ & 60 & -50 & -25 & 5 & 10 & 10 & 14 & 15 & -10 & -3 & 9 & 20 \\
\hline $\mathrm{DA} 48 / 1$ & 49 & -2.5 & 10 & 20 & -19.5 & -5 & -10 & -15.5 & 0 & 5 & -22 & 0 \\
\hline $\mathrm{DA} 48 / 2$ & -15 & -7.5 & 17 & -25.5 & -10 & 1 & -9 & 0 & 1 & 26 & 2 & -10 \\
\hline DA $48 / 3$ & 0 & 5 & 5 & -0.5 & 0 & 9 & -4.5 & -2 & -5 & -5 & -9 & 0 \\
\hline $\mathrm{DA} 48 / 4$ & 59.5 & 17 & 12 & -5 & -16 & 13 & 0 & 1.5 & 2 & 0 & -17.5 & -44.5 \\
\hline $\mathrm{DA} 48 / 5$ & 29.5 & 30 & 10 & 10 & 15 & 15 & 10 & 10 & 9 & 5.5 & 0 & 0 \\
\hline DA $48 / 6$ & 0 & -9 & 0 & -2.5 & -1.5 & 2 & 0 & 0 & 1 & 0 & 0 & 4 \\
\hline DA $68 / 1$ & 40 & 1 & 44 & 20 & 20 & 5 & 15 & 5.5 & 10 & 16 & 17 & 4.5 \\
\hline DA $68 / 2$ & -100 & -90 & -30 & -2.5 & 10 & 30 & 53.5 & 5 & -3 & 0 & 20 & -1 \\
\hline $\mathrm{DA} 68 / 3$ & -10 & -5 & -10 & 20 & 10 & 1.5 & 0 & -4 & 1 & 0 & 2 & -1 \\
\hline $\mathrm{DA} 68 / 4$ & 9 & 0 & 4.5 & -15 & 10 & -2.5 & -2.5 & -7.5 & 5 & 10 & 5 & -5 \\
\hline $\mathrm{DA} 68 / 5$ & 35 & 35 & 5 & 40 & -30 & 10 & 0 & -4.5 & 0 & -10 & 2.5 & 0 \\
\hline $\mathrm{DA} 68 / 6$ & -25 & -12.5 & 0 & 10 & 14 & 0 & -1 & 0 & 0 & 0 & 0 & 0 \\
\hline
\end{tabular}


Table 12: Periodical prices of the risky share

For each condition (CM48, CM68, DA48, DA68) the table reports the periodical prices for risky shares (median prices in the DA treatment, clearing prices in the CM treatment).

\begin{tabular}{|c|c|c|c|c|c|c|c|c|c|c|c|c|}
\hline Period & 1 & 2 & 3 & 4 & 5 & 6 & 7 & 8 & 9 & 10 & 11 & 12 \\
\hline $\mathrm{CM} 48 / 1$ & 211 & 288 & 270 & 294 & 295 & 290 & 287 & 285 & 290 & 280 & 280 & 300 \\
\hline $\mathrm{CM} 48 / 2$ & 150 & 100 & 145 & 160 & 200 & 205 & 225 & 230 & 210 & 231 & 231 & 220 \\
\hline $\mathrm{CM} 48 / 3$ & 300 & 250 & 299 & 300 & 299 & 291 & 292 & 290 & 290 & 293 & 294 & 295 \\
\hline $\mathrm{CM} 48 / 4$ & 200 & 200 & 200 & 180 & 195 & 200 & 210 & 210 & 210 & 210 & 240 & 260 \\
\hline $\mathrm{CM} 48 / 5$ & 500 & 400 & 420 & 300 & 420 & 300 & 300 & 294 & 280 & 300 & 290 & 300 \\
\hline $\mathrm{CM} 48 / 6$ & 200 & 210 & 200 & 210 & 230 & 250 & 240 & 250 & 210 & 250 & 250 & 230 \\
\hline $\mathrm{CM} 68 / 1$ & 170 & 230 & 220 & . & 250 & 250 & 260 & 275 & 280 & 278 & 274 & 275 \\
\hline $\mathrm{CM} 68 / 2$ & 250 & 252 & 255 & 255 & 260 & 260 & 260 & 265 & 265 & 266 & 270 & 271 \\
\hline $\mathrm{CM} 68 / 3$ & 300 & 300 & 300 & 320 & 310 & 330 & 320 & 310 & 305 & 300 & 300 & 300 \\
\hline $\mathrm{CM} 68 / 4$ & 231 & 250 & 249 & 230 & 200 & 230 & 250 & 239 & 249 & 250 & 250 & 250 \\
\hline $\mathrm{CM} 68 / 5$ & 260 & 265 & 255 & 256 & 259 & 270 & 270 & 256 & 270 & 275 & 270 & 270 \\
\hline CM68/6 & 260 & 200 & 190 & 205 & 210 & 230 & 239 & 250 & 240 & 248 & 265 & 265 \\
\hline DA $48 / 1$ & 149 & 150 & 250 & 270 & 220 & 145 & 160 & 195 & 150 & 155 & 200 & 199 \\
\hline DA $48 / 2$ & 250 & 233 & 178 & 175 & 220 & 250 & 240 & 230 & 250 & 250 & 222 & 220 \\
\hline DA $48 / 3$ & 150 & 185 & 190 & 199 & 200 & 219 & 195 & 218 & 220 & 220 & 206 & 210 \\
\hline DA $48 / 4$ & 172 & 200 & 222 & 225 & 200 & 213 & 205 & 200 & 180 & 185 & 170 & 155 \\
\hline DA $48 / 5$ & 180 & 200 & 200 & 210 & 230 & 245 & 240 & 235 & 229 & 225 & 220 & 220 \\
\hline DA $48 / 6$ & 175 & 170 & 170 & 178 & 182 & 195 & 190 & 192 & 192 & 190 & 195 & 199 \\
\hline DA68/1 & 200 & 200 & 239 & 220 & 220 & 215 & 215 & 208 & 220 & 216 & 222 & 220 \\
\hline $\mathrm{DA} 68 / 2$ & 500 & 300 & 260 & 288 & 290 & 290 & 283 & 270 & 267 & 270 & 280 & 278 \\
\hline DA $68 / 3$ & 200 & 195 & 200 & 240 & 240 & 239 & 240 & 241 & 250 & 250 & 255 & 258 \\
\hline DA $68 / 4$ & 199 & 200 & 205 & 215 & 210 & 220 & 223 & 213 & 225 & 210 & 220 & 215 \\
\hline DA $68 / 5$ & 215 & 245 & 250 & 240 & 230 & 270 & 250 & 250 & 260 & 250 & 253 & 260 \\
\hline DA $68 / 6$ & 150 & 163 & 175 & 195 & 199 & 195 & 199 & 200 & 200 & 200 & 200 & 200 \\
\hline
\end{tabular}


Table 13: Periodical prices for the ambiguous share

For each condition (CM48, CM68, DA48, DA68) the table reports the periodical prices for ambiguous shares (median prices in the DA, clearing prices in the $\mathrm{CM}$ treatment).

\begin{tabular}{|c|c|c|c|c|c|c|c|c|c|c|c|c|}
\hline Period & 1 & 2 & 3 & 4 & 5 & 6 & 7 & 8 & 9 & 10 & 11 & 12 \\
\hline $\mathrm{CM} 48 / 1$ & 300 & 293 & 290 & 289 & 288 & 300 & 289 & 285 & 283 & 287 & 280 & 300 \\
\hline $\mathrm{CM} 48 / 2$ & 90 & 160 & 161 & 200 & 225 & 250 & 250 & 230 & 220 & 230 & 230 & 220 \\
\hline $\mathrm{CM} 48 / 3$ & 200 & 299 & 287 & 300 & 290 & 290 & 285 & 290 & 290 & 290 & 293 & 295 \\
\hline $\mathrm{CM} 48 / 4$ & 200 & 200 & 200 & 195 & 200 & 200 & 210 & 210 & 210 & 210 & 225 & 225 \\
\hline $\mathrm{CM} 48 / 5$ & 260 & 400 & 400 & 400 & 350 & 350 & 300 & 299 & 300 & 290 & 305 & 300 \\
\hline $\mathrm{CM} 48 / 6$ & 140 & 210 & 180 & 200 & 200 & 205 & 250 & 180 & 210 & 230 & 220 & 250 \\
\hline $\mathrm{CM} 68 / 1$ & 170 & 180 & 200 & 230 & 230 & 250 & 250 & 260 & 270 & 270 & 280 & 275 \\
\hline $\mathrm{CM} 68 / 2$ & 250 & 240 & 250 & 250 & 260 & 255 & 250 & 260 & 260 & 261 & 262 & 265 \\
\hline $\mathrm{CM} 68 / 3$ & 270 & 290 & 310 & 290 & 290 & 300 & 290 & 290 & 290 & 285 & 285 & 285 \\
\hline $\mathrm{CM} 68 / 4$ & 216 & 231 & 230 & 230 & 200 & 250 & 250 & 235 & 249 & 245 & 249 & 231 \\
\hline $\mathrm{CM} 68 / 5$ & 250 & 250 & 250 & 250 & 256 & 259 & 270 & 269 & 263 & 265 & 256 & 265 \\
\hline $\mathrm{CM} 68 / 6$ & 200 & 250 & 215 & 200 & 200 & 220 & 225 & 235 & 250 & 251 & 256 & 245 \\
\hline DA $48 / 1$ & 100 & 153 & 240 & 250 & 240 & 150 & 170 & 210 & 150 & 150 & 222 & 199 \\
\hline $\mathrm{DA} 48 / 2$ & 265 & 240 & 161 & 200 & 230 & 249 & 249 & 230 & 249 & 224 & 220 & 230 \\
\hline DA $48 / 3$ & 150 & 180 & 185 & 199 & 200 & 210 & 199 & 220 & 225 & 225 & 215 & 210 \\
\hline $\mathrm{DA} 48 / 4$ & 113 & 183 & 210 & 230 & 216 & 200 & 205 & 199 & 178 & 185 & 188 & 200 \\
\hline $\mathrm{DA} 48 / 5$ & 151 & 170 & 190 & 200 & 215 & 230 & 230 & 225 & 220 & 220 & 220 & 220 \\
\hline DA $48 / 6$ & 175 & 179 & 170 & 180 & 184 & 193 & 190 & 192 & 191 & 190 & 195 & 195 \\
\hline DA $68 / 1$ & 160 & 199 & 195 & 200 & 200 & 210 & 200 & 202 & 210 & 200 & 205 & 216 \\
\hline DA68/2 & 600 & 390 & 290 & 290 & 280 & 260 & 230 & 265 & 270 & 270 & 260 & 279 \\
\hline $\mathrm{DA} 68 / 3$ & 210 & 200 & 210 & 220 & 230 & 238 & 240 & 245 & 249 & 250 & 253 & 259 \\
\hline $\mathrm{DA} 68 / 4$ & 190 & 200 & 201 & 230 & 200 & 223 & 225 & 220 & 220 & 200 & 215 & 220 \\
\hline $\mathrm{DA} 68 / 5$ & 180 & 210 & 245 & 200 & 260 & 260 & 250 & 255 & 260 & 260 & 250 & 260 \\
\hline $\mathrm{DA} 68 / 6$ & 175 & 175 & 175 & 185 & 185 & 195 & 200 & 200 & 200 & 200 & 200 & 200 \\
\hline
\end{tabular}


Table 14: Periodical data: differences in volatility

For conditions DA48 and DA68 the table reports the differences in standard deviations of prices between the risky asset $(R)$ and of the ambiguous asset (A) for each period.

\begin{tabular}{|c|c|c|c|c|c|c|c|c|c|c|c|c|}
\hline Period & 1 & 2 & 3 & 4 & 5 & 6 & 7 & 8 & 9 & 10 & 11 & 12 \\
\hline $\mathrm{DA} 48 / 1$ & 6 & 150 & 78 & 3 & -12 & 7 & 1 & 420 & -36 & -10 & -15 & 12 \\
\hline DA $48 / 2$ & 65 & 6 & 56 & -151 & 12 & -8 & -13 & 14 & -2 & -10 & 16 & -17 \\
\hline $\mathrm{DA} 48 / 3$ & 2 & -9 & 3 & -4 & -5 & -288 & 1 & 0 & 1 & 360 & 1 & 0 \\
\hline DA $48 / 4$ & -37 & 11 & 8 & -2 & 1 & -56 & 171 & 1 & -7 & -3 & -7 & 17 \\
\hline DA $48 / 5$ & 45 & 0 & 2 & -6 & 18 & 13 & -1 & 0 & 38 & -118 & 0 & -177 \\
\hline DA $48 / 6$ & 4 & 10 & 0 & 2 & -5 & -3 & -1 & 15 & 0 & 1 & -1 & 1 \\
\hline $\mathrm{DA} 68 / 1$ & 3 & 38 & 24 & -6 & -7 & -46 & 4 & -1 & 43 & -1 & -4 & 29 \\
\hline $\mathrm{DA} 68 / 2$ & -132 & 105 & 171 & -92 & 124 & -169 & 104 & 33 & 3 & -7 & -5 & 0 \\
\hline $\mathrm{DA} 68 / 3$ & 6 & 7 & -1 & 14 & 3 & -25 & 3 & 2 & -1 & -3 & 3 & 1 \\
\hline $\mathrm{DA} 68 / 4$ & -4 & 11 & 10 & 21 & 26 & 25 & 41 & 5 & -11 & -39 & 40 & 2 \\
\hline $\mathrm{DA} 68 / 5$ & -34 & 3 & -18 & -9 & -8 & -4 & -6 & 1 & 2 & -4 & 1 & 15 \\
\hline DA $68 / 6$ & 38 & -2 & -9 & 5 & -5 & -32 & 31 & 17 & 25 & -119 & 39 & -121 \\
\hline
\end{tabular}


Table 15: Periodical data: differences in volume

For each condition (CM48, CM68, DA48, DA68) the table reports the differences in the number of trades between the risky asset $(\mathrm{R})$ and of the ambiguous asset $(\mathrm{A})$ for each period.

\begin{tabular}{|c|c|c|c|c|c|c|c|c|c|c|c|c|}
\hline Period & 1 & 2 & 3 & 4 & 5 & 6 & 7 & 8 & 9 & 10 & 11 & 12 \\
\hline $\mathrm{CM} 48 / 1$ & 1 & -3 & 1 & 0 & 2 & -2 & 1 & 3 & 1 & 4 & 2 & 0 \\
\hline $\mathrm{CM} 48 / 2$ & 2 & -9 & -18 & -8 & -8 & 2 & -5 & 3 & -7 & -1 & -3 & 3 \\
\hline $\mathrm{CM} 48 / 3$ & 8 & -4 & 1 & 0 & 2 & -4 & 0 & 5 & 12 & -5 & 3 & 4 \\
\hline $\mathrm{CM} 48 / 4$ & -2 & -5 & 4 & 1 & -7 & -4 & -2 & -2 & 6 & -7 & 4 & -6 \\
\hline $\mathrm{CM} 48 / 5$ & 2 & 10 & 9 & -2 & 3 & 0 & -1 & -6 & -10 & 3 & 0 & -6 \\
\hline $\mathrm{CM} 48 / 6$ & 4 & 1 & 9 & -10 & 12 & -4 & 4 & 10 & -7 & 0 & 9 & -5 \\
\hline $\mathrm{CM} 68 / 1$ & -4 & -2 & 5 & -18 & -8 & -10 & -4 & -12 & -1 & 0 & -11 & -17 \\
\hline $\mathrm{CM} 68 / 2$ & -4 & 8 & -14 & -18 & 3 & -3 & 1 & 1 & -9 & 7 & -4 & -20 \\
\hline $\mathrm{CM} 68 / 3$ & 5 & 6 & -7 & 13 & -7 & 18 & 22 & 1 & 1 & 3 & -7 & -7 \\
\hline $\mathrm{CM} 68 / 4$ & -8 & -6 & -2 & -2 & 1 & -18 & -5 & -4 & -9 & 1 & -4 & -10 \\
\hline $\mathrm{CM} 68 / 5$ & 0 & -2 & -19 & 0 & -3 & -17 & -6 & -17 & -19 & -2 & -9 & 2 \\
\hline $\mathrm{CM} 68 / 6$ & -7 & 3 & -13 & 5 & 3 & -12 & -9 & -8 & 9 & -2 & 10 & -2 \\
\hline $\mathrm{DA} 48 / 1$ & 13 & 9 & 34 & 15 & -1 & -10 & 1 & -2 & -24 & -4 & 12 & -5 \\
\hline $\mathrm{DA} 48 / 2$ & 3 & 1 & -4 & -2 & 3 & -12 & -10 & -2 & -4 & -10 & 13 & 8 \\
\hline $\mathrm{DA} 48 / 3$ & -15 & 9 & -14 & 1 & 10 & -11 & 2 & 0 & 12 & -2 & 4 & -5 \\
\hline $\mathrm{DA} 48 / 4$ & 0 & -3 & -4 & -10 & 7 & -4 & -5 & 8 & -17 & -4 & 5 & -4 \\
\hline $\mathrm{DA} 48 / 5$ & -3 & -6 & 6 & 0 & -2 & -2 & 16 & -9 & -6 & -10 & -4 & 17 \\
\hline $\mathrm{DA} 48 / 6$ & -4 & 2 & -4 & 9 & 6 & -6 & -2 & -2 & 12 & -3 & -4 & 15 \\
\hline $\mathrm{DA} 68 / 1$ & 7 & 0 & 5 & -6 & -14 & 11 & -1 & 7 & -3 & 7 & 2 & 17 \\
\hline $\mathrm{DA} 68 / 2$ & 7 & 8 & 10 & -6 & -7 & -12 & 6 & 8 & -4 & -7 & 4 & 7 \\
\hline $\mathrm{DA} 68 / 3$ & -3 & -14 & 8 & -3 & -5 & -4 & -8 & -4 & -19 & -24 & -14 & -5 \\
\hline $\mathrm{DA} 68 / 4$ & 12 & 7 & 10 & -1 & 16 & 15 & 13 & -7 & -5 & -14 & 7 & 6 \\
\hline $\mathrm{DA} 68 / 5$ & -9 & 18 & -7 & -27 & -24 & -13 & -2 & -18 & 5 & -23 & -9 & -9 \\
\hline DA $68 / 6$ & 5 & 7 & -1 & -4 & -5 & 0 & 29 & 22 & 7 & 7 & 19 & 10 \\
\hline
\end{tabular}


Table 16: Executed trades in the risky market

For each condition (CM48, CM68, DA48, DA68) the table reports the number of trades for risky shares.

\begin{tabular}{|c|c|c|c|c|c|c|c|c|c|c|c|c|}
\hline Period & 1 & 2 & 3 & 4 & 5 & 6 & 7 & 8 & 9 & 10 & 11 & 12 \\
\hline $\mathrm{CM} 48 / 1$ & 12 & 12 & 6 & 8 & 9 & 9 & 5 & 11 & 11 & 7 & 11 & 9 \\
\hline $\mathrm{CM} 48 / 2$ & 16 & 17 & 10 & 14 & 11 & 20 & 20 & 17 & 15 & 30 & 18 & 13 \\
\hline $\mathrm{CM} 48 / 3$ & 14 & 6 & 15 & 13 & 13 & 11 & 15 & 16 & 20 & 10 & 14 & 21 \\
\hline $\mathrm{CM} 48 / 4$ & 3 & 5 & 10 & 10 & 6 & 16 & 15 & 11 & 15 & 14 & 12 & 12 \\
\hline $\mathrm{CM} 48 / 5$ & 10 & 21 & 21 & 15 & 14 & 15 & 15 & 9 & 13 & 21 & 20 & 20 \\
\hline $\mathrm{CM} 48 / 6$ & 21 & 17 & 18 & 15 & 22 & 15 & 20 & 18 & 9 & 15 & 27 & 10 \\
\hline $\mathrm{CM} 68 / 1$ & 11 & 10 & 19 & 0 & 14 & 7 & 7 & 4 & 5 & 17 & 16 & 16 \\
\hline $\mathrm{CM} 68 / 2$ & 16 & 18 & 16 & 9 & 14 & 10 & 11 & 20 & 10 & 20 & 15 & 14 \\
\hline $\mathrm{CM} 68 / 3$ & 11 & 15 & 3 & 22 & 14 & 24 & 28 & 20 & 10 & 19 & 10 & 19 \\
\hline $\mathrm{CM} 68 / 4$ & 12 & 5 & 13 & 8 & 8 & 8 & 6 & 2 & 10 & 11 & 6 & 10 \\
\hline $\mathrm{CM} 68 / 5$ & 12 & 6 & 6 & 10 & 13 & 13 & 20 & 13 & 16 & 20 & 16 & 30 \\
\hline $\mathrm{CM} 68 / 6$ & 13 & 18 & 10 & 25 & 25 & 10 & 16 & 11 & 22 & 17 & 16 & 18 \\
\hline $\mathrm{DA} 48 / 1$ & 35 & 51 & 73 & 59 & 49 & 29 & 53 & 44 & 35 & 40 & 45 & 39 \\
\hline $\mathrm{DA} 48 / 2$ & 12 & 30 & 17 & 24 & 37 & 31 & 35 & 36 & 35 & 28 & 37 & 42 \\
\hline $\mathrm{DA} 48 / 3$ & 34 & 45 & 35 & 36 & 43 & 24 & 28 & 29 & 34 & 24 & 31 & 29 \\
\hline $\mathrm{DA} 48 / 4$ & 18 & 21 & 13 & 19 & 23 & 19 & 24 & 22 & 4 & 13 & 13 & 8 \\
\hline $\mathrm{DA} 48 / 5$ & 29 & 31 & 33 & 39 & 32 & 30 & 48 & 25 & 25 & 26 & 25 & 35 \\
\hline DA $48 / 6$ & 5 & 13 & 12 & 18 & 24 & 9 & 27 & 22 & 31 & 26 & 21 & 42 \\
\hline $\mathrm{DA} 68 / 1$ & 23 & 27 & 42 & 31 & 26 & 36 & 20 & 24 & 19 & 19 & 21 & 25 \\
\hline $\mathrm{DA} 68 / 2$ & 27 & 27 & 31 & 28 & 23 & 22 & 30 & 35 & 28 & 19 & 31 & 33 \\
\hline DA $68 / 3$ & 20 & 12 & 30 & 17 & 21 & 24 & 24 & 26 & 22 & 21 & 21 & 30 \\
\hline DA $68 / 4$ & 23 & 17 & 22 & 23 & 34 & 27 & 32 & 22 & 14 & 18 & 23 & 22 \\
\hline DA68 $/ 5$ & 16 & 28 & 25 & 37 & 14 & 29 & 41 & 38 & 35 & 29 & 32 & 29 \\
\hline DA $68 / 6$ & 20 & 28 & 31 & 39 & 35 & 31 & 47 & 47 & 33 & 37 & 48 & 33 \\
\hline
\end{tabular}


Table 17: Executed trades in the ambiguous market

For each condition (CM48, CM68, DA48, DA68) the table reports the number of trades for ambiguous shares.

\begin{tabular}{|c|c|c|c|c|c|c|c|c|c|c|c|c|}
\hline Period & 1 & 2 & 3 & 4 & 5 & 6 & 7 & 8 & 9 & 10 & 11 & 12 \\
\hline $\mathrm{CM} 48 / 1$ & 11 & 15 & 5 & 8 & 7 & 11 & 4 & 8 & 10 & 3 & 9 & 9 \\
\hline $\mathrm{CM} 48 / 2$ & 14 & 26 & 28 & 22 & 19 & 18 & 25 & 14 & 22 & 31 & 21 & 10 \\
\hline $\mathrm{CM} 48 / 3$ & 6 & 10 & 14 & 13 & 11 & 15 & 15 & 11 & 8 & 15 & 11 & 17 \\
\hline $\mathrm{CM} 48 / 4$ & 5 & 10 & 6 & 9 & 13 & 20 & 17 & 13 & 9 & 21 & 8 & 18 \\
\hline $\mathrm{CM} 48 / 5$ & 8 & 11 & 12 & 17 & 11 & 15 & 16 & 15 & 23 & 18 & 20 & 26 \\
\hline $\mathrm{CM} 48 / 6$ & 17 & 16 & 9 & 25 & 10 & 19 & 16 & 8 & 16 & 15 & 18 & 15 \\
\hline $\mathrm{CM} 68 / 1$ & 15 & 12 & 14 & 18 & 22 & 17 & 11 & 16 & 6 & 17 & 27 & 33 \\
\hline $\mathrm{CM} 68 / 2$ & 20 & 10 & 30 & 27 & 11 & 13 & 10 & 19 & 19 & 13 & 19 & 34 \\
\hline $\mathrm{CM} 68 / 3$ & 6 & 9 & 10 & 9 & 21 & 6 & 6 & 19 & 9 & 16 & 17 & 26 \\
\hline $\mathrm{CM} 68 / 4$ & 20 & 11 & 15 & 10 & 7 & 26 & 11 & 6 & 19 & 10 & 10 & 20 \\
\hline $\mathrm{CM} 68 / 5$ & 12 & 8 & 25 & 10 & 16 & 30 & 26 & 30 & 35 & 22 & 25 & 28 \\
\hline $\mathrm{CM} 68 / 6$ & 20 & 15 & 23 & 20 & 22 & 22 & 25 & 19 & 13 & 19 & 6 & 20 \\
\hline DA $48 / 1$ & 22 & 42 & 39 & 44 & 50 & 39 & 52 & 46 & 59 & 44 & 33 & 44 \\
\hline DA $48 / 2$ & 9 & 29 & 21 & 26 & 34 & 43 & 45 & 38 & 39 & 38 & 24 & 34 \\
\hline DA $48 / 3$ & 49 & 36 & 49 & 35 & 33 & 35 & 26 & 29 & 22 & 26 & 27 & 34 \\
\hline DA $48 / 4$ & 18 & 24 & 17 & 29 & 16 & 23 & 29 & 14 & 21 & 17 & 8 & 12 \\
\hline DA $48 / 5$ & 32 & 37 & 27 & 39 & 34 & 32 & 32 & 34 & 31 & 36 & 29 & 18 \\
\hline DA48/6 & 9 & 11 & 16 & 9 & 18 & 15 & 29 & 24 & 19 & 29 & 25 & 27 \\
\hline DA68/1 & 16 & 27 & 37 & 37 & 40 & 25 & 21 & 17 & 22 & 12 & 19 & 8 \\
\hline DA68 $/ 2$ & 20 & 19 & 21 & 34 & 30 & 34 & 24 & 27 & 32 & 26 & 27 & 26 \\
\hline DA68/3 & 23 & 26 & 22 & 20 & 26 & 28 & 32 & 30 & 41 & 45 & 35 & 35 \\
\hline DA68/4 & 11 & 10 & 12 & 24 & 18 & 12 & 19 & 29 & 19 & 32 & 16 & 16 \\
\hline DA68/5 & 25 & 10 & 32 & 64 & 38 & 42 & 43 & 56 & 30 & 52 & 41 & 38 \\
\hline DA68/6 & 15 & 21 & 32 & 43 & 40 & 31 & 18 & 25 & 26 & 30 & 29 & 23 \\
\hline
\end{tabular}


Table 18: Periodical data of differences in dispersion of shares

For each condition (CM48, CM68, DA48, DA68) the table reports the differences in the standard deviation of end-of-period-stock holdings between the risky asset $(R)$ and of the ambiguous asset (A) for each period.

\begin{tabular}{|c|c|c|c|c|c|c|c|c|c|c|c|c|}
\hline Period & 1 & 2 & 3 & 4 & 5 & 6 & 7 & 8 & 9 & 10 & 11 & 12 \\
\hline $\mathrm{CM} 48 / 1$ & -1.22 & -1.14 & 0.79 & 1.14 & -0.64 & -1.23 & 0.05 & 0.61 & 1.72 & 0.65 & 0.95 & 2.12 \\
\hline $\mathrm{CM} 48 / 2$ & 0.86 & -4.55 & -3.42 & -1.13 & -1.75 & 2.11 & -1.49 & 0.80 & -3.89 & -0.09 & -1.20 & -0.03 \\
\hline $\mathrm{CM} 48 / 3$ & 0.32 & 0.74 & -0.86 & -0.74 & -0.27 & 0.48 & -0.99 & 2.22 & 1.03 & -1.08 & 0.24 & -0.53 \\
\hline $\mathrm{CM} 48 / 4$ & -1.09 & -2.33 & -0.55 & 0.38 & 0.80 & -1.59 & -2.35 & -1.92 & 0.30 & -0.69 & 1.39 & -1.06 \\
\hline $\mathrm{CM} 48 / 5$ & 0.64 & 1.46 & 1.97 & -2.15 & 2.36 & -0.37 & 0.82 & -3.28 & -3.40 & 1.21 & -1.26 & -1.86 \\
\hline $\mathrm{CM} 48 / 6$ & 2.16 & -2.74 & 2.22 & -3.12 & 3.54 & -2.45 & 0.22 & 2.94 & -1.87 & 0.71 & 3.00 & -2.19 \\
\hline $\mathrm{CM} 68 / 1$ & -0.79 & -0.57 & 0.85 & -6.81 & -1.95 & -2.29 & -1.79 & -2.25 & -0.35 & -0.52 & -2.08 & -2.83 \\
\hline $\mathrm{CM} 68 / 2$ & -0.65 & 2.97 & -3.20 & -4.56 & 2.02 & -1.07 & -0.12 & 0.33 & -2.77 & 0.14 & 0.41 & -2.58 \\
\hline $\mathrm{CM} 68 / 3$ & 3.08 & 0.15 & -2.48 & 3.37 & -2.29 & 5.00 & 5.86 & 1.09 & 0.03 & -1.78 & -2.25 & -2.78 \\
\hline $\mathrm{CM} 68 / 4$ & -2.38 & 0.14 & 1.05 & -1.54 & -0.27 & -4.30 & -0.43 & -1.03 & -2.50 & -2.11 & -0.55 & -3.34 \\
\hline CM68/5 & 1.75 & -1.65 & -3.83 & 1.32 & 0.78 & -1.46 & -1.67 & -3.16 & -2.25 & -2.88 & -1.13 & 0.58 \\
\hline CM68/6 & -5.33 & 2.27 & -4.40 & -0.84 & 1.15 & -4.44 & -1.46 & -4.79 & -0.76 & 2.06 & 2.47 & -0.60 \\
\hline $\mathrm{DA} 48 / 1$ & 0.48 & -1.96 & 0.40 & -0.74 & 1.27 & 1.55 & -2.92 & -3.17 & 0.10 & 0.58 & 0.98 & -0.03 \\
\hline $\mathrm{DA} 48 / 2$ & -0.88 & -0.09 & -0.19 & 1.95 & 1.86 & 0.87 & 1.19 & -0.26 & 2.15 & -1.09 & -0.93 & 0.42 \\
\hline DA $48 / 3$ & -2.44 & -1.10 & -1.27 & 0.33 & 2.98 & 3.04 & 2.37 & -0.38 & 0.22 & 0.33 & -0.79 & 0.93 \\
\hline DA $48 / 4$ & 0.89 & 0.75 & -1.77 & -3.15 & 1.81 & 1.47 & -2.33 & 2.82 & -3.78 & 0.40 & 1.75 & -3.73 \\
\hline $\mathrm{DA} 48 / 5$ & 0.00 & 4.06 & 1.36 & -3.06 & 1.84 & -1.48 & -0.82 & 0.41 & 2.08 & -0.40 & 0.03 & 0.93 \\
\hline DA $48 / 6$ & 0.76 & -0.42 & -0.93 & 1.54 & -1.53 & -1.72 & -0.56 & -1.48 & -0.70 & -1.93 & -0.09 & 0.88 \\
\hline DA68/1 & 0.30 & -0.85 & 2.83 & 2.83 & 0.82 & 0.93 & 0.88 & 0.26 & -1.93 & -1.18 & -0.84 & 0.38 \\
\hline DA $68 / 2$ & 1.65 & -1.71 & -0.71 & -0.66 & 1.23 & 1.56 & 3.44 & -0.27 & -4.02 & -1.54 & 0.33 & 0.91 \\
\hline DA $68 / 3$ & -0.17 & -0.61 & -0.42 & -1.42 & 1.02 & -0.68 & -0.38 & -2.70 & -1.61 & -3.38 & -3.55 & -1.27 \\
\hline DA $68 / 4$ & 2.00 & -0.08 & 2.69 & 0.66 & -0.65 & 1.63 & 2.54 & -3.63 & -2.83 & -1.80 & 1.61 & 0.23 \\
\hline DA68/5 & 1.04 & 0.21 & -0.13 & -0.21 & -0.70 & -1.04 & 1.69 & -0.74 & -0.88 & -2.89 & -2.49 & -3.10 \\
\hline DA $68 / 6$ & 0.50 & -0.36 & 2.14 & -1.44 & 2.01 & -1.09 & 0.33 & 3.01 & -1.68 & -0.40 & 1.72 & 0.98 \\
\hline
\end{tabular}




\section{E Sample of instructions}

Translated from German and formatted in a more compact way than in the actual handouts for the subjects. Note that participants saw the trading screen and interacted with it while the instructions were being read out aloud. Sections 2,3 , and 4 of the instructions only differ in the number of winning colors. Section 1 of the instructions (Trading in the market) explains the market institution. A complete set of instructions for DM48 can be found in section E.1. Furthermore, we provide the instructions for the market instructions of the CM in treatment in section E.2. The instructions for the call market are basically taken from Cheung and Palan (2012).

\section{E.1 Instructions DA48}

Welcome to the experiment. You are participating in this experiment to earn money. Accordingly, you should try to maximize the payoff in this experiment. If you follow the instructions carefully, and if your decisions are good, you will earn a significant amount of money. In this experiment you trade shares. All transactions are calculated in "Taler." At the end of the experiment your total amount earned in Talers will be exchanged and paid to you at the following exchange rate: 200 Talers $=1$ Euro.

\section{Trading in the market}

The trading of shares will be on a market platform. Thus, you first have to gain some experience with the trading platform. For the trading actions you need Talers and shares. You find your inventory in Talers and shares in the field below the trading platform.

If you want to sell a share, you can use the text box "ENTER SELLING PRICE." In this text box you enter the price at which you are willing to sell the share. Afterwards you have to click on the "SUBMIT" button below the text box. Please do this right now (you can type in any arbitrary price)! Once you have done this, you will recognize that there are eight prices (one of each participant) recorded in the schedule named "QUOTES TO SELL" on the left-hand side. The lowest quote is listed in the first row and it will be emphasized. If you click the "BUY" button you will buy a share at the current lowest selling price. If you first select another price of the list by clicking on it, you will buy the share at the selected price. Please buy a share now by clicking on it and subsequently press the "BUY" button. Now, everybody should own the same amount of shares as in the beginning, because all of you have offered one share to sell and at the same time all of you have purchased one share.

If you want to buy a share, please use the text box "ENTER BUYING PRICE." In this text box you enter the price at which you are willing to buy the share. Afterwards, click on the "SUBMIT" button which you can find below the text box. Please, do this right now (you can type in any arbitrary price)! Once you have done this, you will 
recognize that there are eight prices (one of each participant) recorded in the schedule named "QUOTES TO BUY" on the right-hand side. The highest quote is listed in the first row and it is emphasized. If you click the "SELL" button you will sell a share at the current highest buying price. If you first select another price on the list by clicking on it, you will sell the share at the selected price. Please sell a share now by clicking on a price and then on the "SELL" button. Now, everybody should own the same amount of shares as in the beginning, because all of you have bought one share and all of you have sold one share.

Remember: if you buy a share, your income is reduced by the purchase price times the number of shares bought. At the same time the amount of shares owned increases. If you sell a share, your income increases by the purchase price times the number of shares bought and your amount of shares owned decreases. The corresponding prices will be recorded in the list "PRICES." The sequence of the prices will depend on the point in time when the prices are set.

At first there will be a two-minutes trial period. The duration of the period will be displayed in the upper-right-hand corner of the screen. None of your actions in the trial period will affect your payoff. Thus, these actions will not impact your starting position in the experiment. The only goal is to gain experience with the trading platform. Make sure that you have successfully submitted bids to buy and to sell stocks. Furthermore ensure that you have at least accepted one offer to buy and one offer to sell. Please feel free to ask questions during the trial period. Thereafter (in subsequent periods) you will always be given the possibility to ask questions. Keep in mind: the better you understand the trading platform, the more you can focus on other important aspects of the experiment.

\section{Procedure in one period}

In each period you will trade two types of shares in two separate markets: share BLUE and share YELLOW (this is different from the trial period). The markets will be open for exactly four minutes. At the beginning of each period you will be given a separate endowment of shares and Talers. At the end of the period all the shares you own will pay out either 300 or 124 Talers each. This payout will be randomly determined (see Section 3 for more detailed information ). At the end of each period you will be able to see the computation of the period's payoff. In total there will be 12 periods.

\section{Share payoff}

After each period the payoffs of the two shares will be determined. Therefore a bag filled with marbles will be provided for each share.

Share BLUE: For share BLUE every bag will contain 8 marbles with 8 different colors (white, red, purple, blue, black, brown, bright green, dark green).

Share YELLOW: Each of the bags might contain 1 to 8 different colors (white, red, purple, blue, black, brown, bright green, dark green). There will be a different bag for each period, i.e., a total of 12 different bags. All color combinations are possible for 
each bag. Thus, it might happen that one bag contains exactly 8 marbles of the same color. However, another possibility is that there is one bag which contains exactly one marble of all 8 colors. All combinations between these two examples might are also possible.

As you have realized, the bags for the share YELLOW were composed by four randomly selected persons at the beginning of the experiment. Each of these persons received three empty bags and were asked to pick eight marbles of almost eight colors. Thus, these persons had the possibility to fill all of these bags with an arbitrary composition of the eight colors. Afterwards the bags were closed. Thus, neither you nor the experimenters know the composition of the marbles in the bags. To ensure that there are exactly eight marbles in each of the bags, the experimenter will count the marbles through the fabric of the bags. You can also check this after the experiment has been finished if you want. The persons who have composed these bags will not take part in the experiment. In total there are 12 bags (three bags filled by each of the four persons) which will be randomly assigned to the 12 periods before we start with the trading.

After each period, each share's value will be determined by the following procedure: At the beginning of the experiment, you marked four colors for the BLUE share and four colors for the YELLOW share. The selections of colors of the whole group (12 for share BLUE and 12 for share YELLOW) will be randomly assigned to the 12 periods. After each period, one participant will draw exactly one marble out of the 'BLUE bag' which will be used. The drawn marble will be used to determine the payout of the BLUE share. Furthermore, another participant will also draw exactly one marble out of the 'YELLOW bag' which was assigned to that period. The drawn marble will be used to determine the payout of the YELLOW share. For both shares we apply the following payout procedure: If the drawn marble's color for the BLUE (YELLOW) share equals one of the four marked colors, then the share's payout is 300 Talers. Otherwise the payout is 124 Talers. The values will be entered in the experimenter computer by the supervisor and it will be shown on your screen.

\section{Endowment and payoff}

At the beginning of each period you will get an endowment as depicted in the table below. Four participants will receive endowment type I. Furthermore, four participants will receive endowment type II. These types will be randomly assigned by the computer at the beginning of each period. At the end of each period you will have to return 3,000 Talers.

\begin{tabular}{|c|c|c|c|c|}
\cline { 2 - 5 } \multicolumn{1}{c|}{} & \multicolumn{2}{c|}{ Blue market } & \multicolumn{2}{c|}{ Yellow market } \\
\hline $\begin{array}{c}\text { endowment } \\
\text { type }\end{array}$ & $\begin{array}{c}\text { number } \\
\text { of shares }\end{array}$ & $\begin{array}{c}\text { available } \\
\text { Talers }\end{array}$ & $\begin{array}{c}\text { number } \\
\text { of shares }\end{array}$ & $\begin{array}{c}\text { available } \\
\text { Talers }\end{array}$ \\
\hline \hline I & 10 & 4,200 & 6 & 5,100 \\
\hline \hline II & 6 & 5,100 & 10 & 4,200 \\
\hline
\end{tabular}


Talers in the BLUE market can only be used for trading in the BLUE market. At the same time Talers in the YELLOW market can only be used for trading in the YELLOW market. At the end of each period, your total payoff (in Talers) is calculated as: available Talers at the beginning of a period + amount of shares $\times$ share payoff (300 or 124) + Talers earned by selling shares - Talers spent on buying shares - return of 3000 Talers.

At the end of the experiment, only one period will be paid out in cash. This period will be determined by a throw of a 12-sided dice. Furthermore, only one market (BLUE or YELLOW) will be paid out. This market will be determined by a coin toss. Thus, there is exactly one period (out of all 12 possible periods) of exactly one market (of share BLUE or YELLOW) which will be paid out to you. Keep in mind that every decision for each share in each period might be essential for your actual payment! You receive an additional show-up fee of 4 Euros for your participation in the experiment. These 4 Euros will be added to your cash payment. The final payment in cash is determined as: Payment (in Euro) $=$ Taler payoff $/ 200+4$ Euros (show-up fee).

\section{E.2 Instructions CM: 1. Trading in the market}

The trading of the assets will be on a market platform. Thus, you first have to gain experience with the trading platform. For the trading actions you need Talers and shares. You find your inventory in Talers and shares in the field below the trading platform. In each period, you will see four input areas to buy and sell the assets.

If you want to sell shares, enter the number of shares you are willing to sell in the field: "Number of shares to sell." Afterwards enter the lowest price you are willing to sell the shares. If you want to buy shares then you make purchase offers by entering the number of shares you are willing to buy in the field "Number of shares to buy." Afterwards enter the highest price you are willing to pay for the shares.

In each period you have the possibility to post sell and buy offers. The price you are willing to buy must be below the price you are willing to sell. The price you post corresponds to one share. At the same time if you sell shares you will get the selling price $\times$ number of sold shares. If you buy shares you will get: the purchase price $\times$ number of bought shares. As soon as you click the OK button, the sell and buy offers will be submitted and cannot be changed anymore.

The computer applies the sell and buy offers of all market participants to determine the market price. All transactions in this period will be processed at this price. This price will be chosen such that the number of shares where sell offers were below or equal this price is of the same number as of the shares where buy offers where higher or equal this price.

The market participants who submitted buy offers which are higher than this price, will buy at this price. Their money endowment will be reduced by the product of market price $\mathrm{x}$ number of shares bought. Furthermore their asset endowment will be increased by the number of assets bought. The market 
participants who submitted sell offers below this price will sell at this price. Their money endowment will be increased by the product of market price $\mathrm{x}$ number of shares sold. Furthermore, their asset endowment will be decreased by the number of shares sold.

In the following you have to launch sell and buy offers for trial purposes. None of these practicing actions will influence your payoffs in this experiment. This is why it will not affect your position later in the experiment. Here, the goal is to familiarize you with the platform only. Therefore you have to launch sell and buy offers three times in a row. Afterwards there will be a brief clearing each time. Feel free to ask questions during the trial period. Afterwards (in later periods) you will always have the possibility to ask questions. Keep in mind, the better you understand the platform, the more you can concentrate on the essential parts of the experiment. 